\title{
ArcheoSciences
}

Revue d'archéométrie

\section{Échanges transmanche de traditions techniques dans la préparation des céramiques : le site portuaire gaulois de la Batterie-Basse à Urville- Nacqueville (Manche)}

Cross-channel exchanges of technical traditions in the ceramics preparation: the Gallic port site of La Batterie-Basse at Urville-Nacqueville (Manche)

\section{Benjamin Gehres et Anthony Lefort}

\section{(2) OpenEdition}

Journals

Édition électronique

URL : https://journals.openedition.org/archeosciences/5274

DOI : $10.4000 /$ archeosciences.5274

ISSN : 2104-3728

Éditeur

Presses universitaires de Rennes

Édition imprimée

Date de publication : 27 juin 2018

Pagination : $57-74$

ISBN : 978-2-7535-7587-5

ISSN : $1960-1360$

\section{Référence électronique}

Benjamin Gehres et Anthony Lefort, «Échanges transmanche de traditions techniques dans la préparation des céramiques : le site portuaire gaulois de la Batterie-Basse à Urville-Nacqueville (Manche) », ArcheoSciences [En ligne], 42-1 | 2018, mis en ligne le 27 juin 2020, consulté le 01 février 2022. URL : http://journals.openedition.org/archeosciences/5274 ; DOI : https://doi.org/10.4000/ archeosciences.5274 


\title{
Échanges transmanche de traditions techniques dans la préparation des céramiques : le site portuaire gaulois de la Batterie-Basse à Urville-Nacqueville (Manche)
}

\author{
Cross-channel Exchanges of Technical Traditions in the Ceramics Preparation: \\ The Gallic Port Site of La Batterie-Basse at Urville-Nacqueville (Manche)
}

\author{
Benjamin GeHres ${ }^{a}$ \\ avec la collaboration de Anthony LEFORT ${ }^{b}$
}

\begin{abstract}
Résumé : L'étude pétrographique et géochimique de céramiques découvertes dans la zone atelier de fabrication d'anneaux en lignite du site portuaire de la Batterie-Basse à Urville-Nacqueville (Manche) a permis d'observer l'utilisation de plusieurs matières premières régionales pour façonner les poteries du site. De plus, l'importation de terres cuites a pu être mise en avant, depuis la zone atelier de Saint-Jean-du-Doigt (Finistère) à plus de $180 \mathrm{~km}$ et depuis la région de la plaine de Caen. Un groupe de pâte domine largement le corpus, caractérisé par une tradition technique particulière dans la préparation des pâtes des terres cuites, le rajout de coquilles broyées aux terres. En effet, des analyses pétrographiques, complétées par une approche par spectrométrie de masse à source plasma, couplée à un système d'ablation laser des inclusions bioclastiques, ont démontré que ce dégraissant ne correspond pas à des fossiles présents naturellement dans les argiles, mais que les potiers ont ajouté à leurs matières premières des fragments de coquilles préalablement broyées. Il s'agit d'une tradition qui n'est pour l'instant pas connue autre part au second âge du Fer dans l'Ouest de la France, et il faut se tourner vers les côtes anglaises pour identifier des procédés similaires. Il en va de même pour plusieurs autres pratiques observées sur le site de la Batterie-Basse, dont les racines pourraient se trouver de l'autre côté de la Manche.
\end{abstract}

Abstract: The petrographic and geochemical studies of ceramics found in the workshop area of the site of La Batterie-Basse at Urville-Nacqueville (Manche, France) allowed us to observe that potters have used several regional raw materials to shape their ceramics. Furthermore, importations of pottery coming from the workshop area of Saint-Jean-du-Doigt (Finistère, France) at $180 \mathrm{~km}$ away and from the region of the "Plaine de Caen" have been highlighted. One type of paste characterised by a particular tradition in the preparation of the clay dominates the corpus, the adjunction of crushed shells. Indeed, the petrographic analysis, completed by a new approach by LA-ICP-MS of the biomineral inclusions, have shown that this temper does not correspond to a naturally present fossil temper, but to a voluntary adjunction by the potters. This tradition is unknown elsewhere in the Late Iron Age in western France, and we must turn to the English coasts to identify similar processes. The same goes for several other practices observed on the site of La Batterie-Basse, whose roots could be on the other side of the English Channel.

Mots clés : Second âge du Fer, Échanges transmanche, Traditions techniques, Céramiques, Pétrographie, LA-ICP-MS.

Keywords: Late Iron age, Cross-Channel exchanges, Traditional technic, Ceramics, Petrography, LA-ICP-MS.

${ }^{a}$ CReAAH-UMR 6566, Laboratoire Archéosciences, Rennes; CEMEF-CNRS UMR 7635/MINES ParisTech, 1 rue Claude-Daunesse CS 10207, 06904 SOPHIA ANTIPOLIS. (mailto:benjamin.gehres@gmail.com)

${ }^{b} 25$ ter Ertot, 50340 LES PIEUX. (lefort.anthony@sfr.fr) 


\section{INTRODUCTION}

Le site portuaire de la Batterie-Basse se développe à partir du second âge du Fer, sur le littoral normand, dans la région d'Urville-Nacqueville (fig. 1), au nord du Cotentin (Manche). Le choix d'implantation de cette occupation n'est pas anodin puisqu'il se trouve au débouché d'une voie de communication entre le sud de l'Angleterre et le réseau fluviatile de la Seine conférant ainsi aux habitants la possibilité d'être au centre des réseaux d'échanges et d'accéder à des produits provenant de plus ou moins longues distances, comme de l'ambre de la Baltique et des amphores italiques (Lefort et al., 2015).

Une répartition spatiale des activités est attestée, comprenant une zone d'activités artisanales, destinée entre autres au façonnage de bracelets en lignite du Dorset (Giot et al., 1986; Baron, 2012, Lefort et al., 2015) et une nécropole avec notamment des restes de banquets funéraires et de bûchers (Lefort et al., 2015). Les premières traces d'occupation datent du II ${ }^{\mathrm{e}}$ siècle av. n.è., soit à La Tène C2-D1, selon les découvertes faites sur le site (monnaies, fragment de bracelet en verre, amphores Dressel 1 a). L'absence d'amphore du type Dressel $1 \mathrm{~b}$ serait un argument plaidant en faveur d'un déclin du site vers La Tène D1b ou au début de La Tène D2 (début du Ir siècle av. n.è.; Lefort, 2012) et d'un abandon durant le $\mathrm{I}^{\mathrm{er}}$ siècle av. n.è. (Lefort, 2012; Lefort et al., 2015), pour être réoccupé durant le Haut-Empire (Lefort et al., 2015).

Le site de la Batterie-Basse se trouve à quelque $110 \mathrm{~km}$ des côtes anglaises et de l'oppidum portuaire d'Hengistbury Head, carrefour d'échanges entre la Gaule armoricaine et l'Angleterre (Cunliffe et al., 1987).

On soulignera l'existence dans la région de précédentes études pétrographiques de céramiques. Ainsi, en plaine de
Caen (fig. 1), des céramiques dites "productions de la plaine de Caen " (fig. 2) sont caractérisées par des pâtes bioclastiques facilement identifiables à la loupe. Ces inclusions sont composées de fragments grossiers d'organismes marins fossiles mal triés : huîtres, brachiopodes, bryozoaires, échinodermes, crinoïdes, ainsi que des fragments de calcaire coquillier. Ces pâtes sont présentes dès l'âge du Bronze et deviennent très courantes à la fin de l'âge du Fer, où elles sont associées à un large éventail typologique, des poteries hautes de stockage à des formes plus petites à basses (Manson et al., 2011). Ces productions sont bien connues depuis les nombreux travaux réalisés par G. San Juan, X. Savary et A.-L. Manson (San Juan et al., 1999 a, 1999 b; Carpentier et al., 2002; Besnard-Vauterin et al., 2009; Jahier et al., 2011; Manson et al., 2011). Elles ont été retrouvées sur des sites implantés dans les secteurs du Bessin, des campagnes de Caen et de Falaise et jusqu'à la bordure occidentale du Pays d'Auge (fig. 2). Ces différentes observations ont ainsi motivé la réalisation d'une étude archéométrique des céramiques découvertes dans la zone artisanale d'Urville-Nacqueville, dans le but de déceler la présence d'importations techniques ou matérielles.

Enfin, sur le massif Armoricain deux ateliers de potiers utilisant les produits d'altérations de roches gabbrö̈ques sont connus. Ces zones de productions n'ont, à ce jour, pas été découvertes sur le terrain, et sont uniquement caractérisées à partir des études pétrographiques des céramiques. Ainsi, la répartition des nombreuses découvertes autour de deux massifs gabbroïques a amené les chercheurs à supposer l'existence de deux zones de productions de poteries dans ces régions. L'une est située à Trégomar, dans les Côtes-d'Armor (Morzadec, 1995) et l'autre, à Saint-Jean-du-Doigt, dans le Finistère (Giot et al., 1986; Giot et Querré, 1987). Ces vases ont connu une diffusion importante, et peuvent être

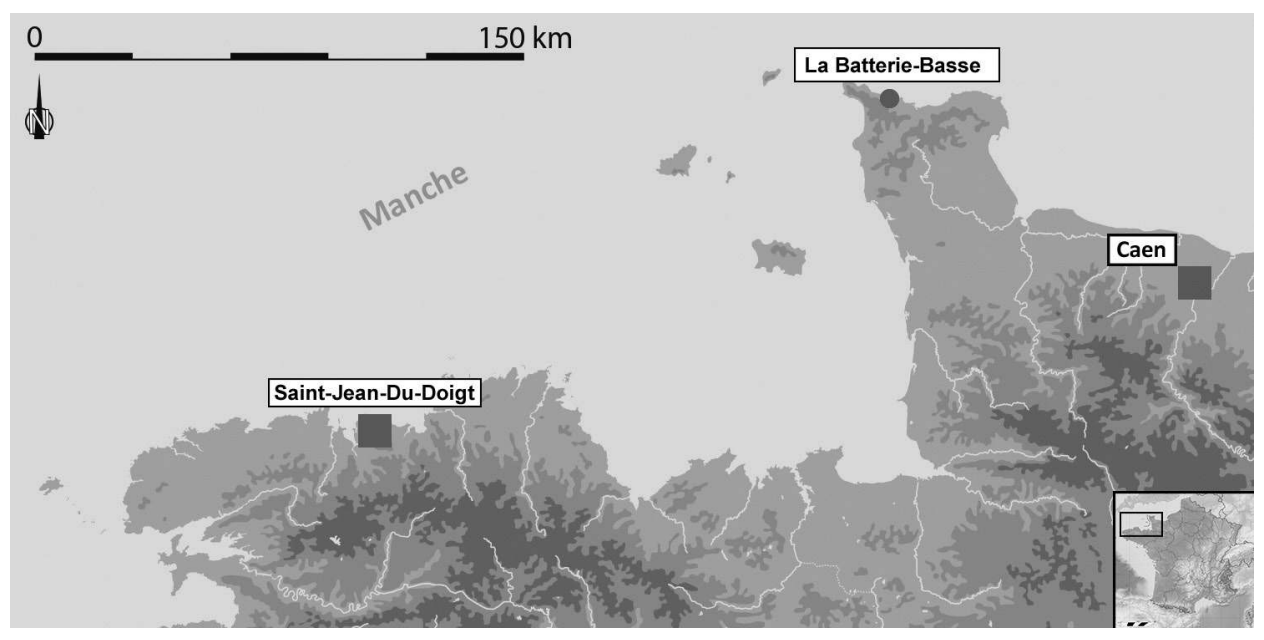

Figure 1 : Localisation du site de la Batterie-Basse et des principales zones d'importations de céramiques.

Figure 1: Localisation of the site of La Batterie-Basse and his principal areas of ceramic importations. 


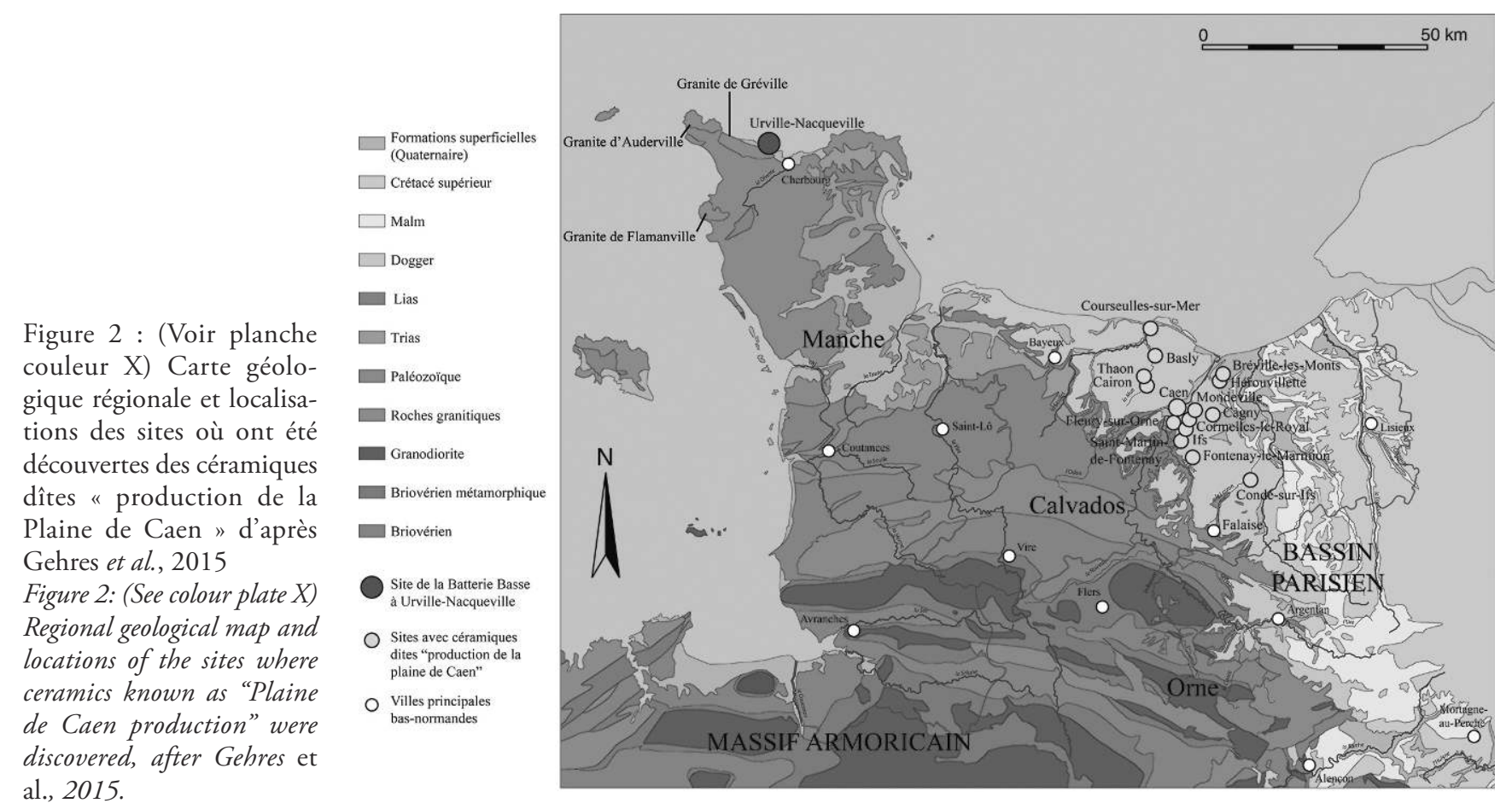

retrouvés sur des sites éloignés de plusieurs centaines de kilomètres, dont le port d'Hengistbury Head en Angleterre (Morzadec, 1995).

Ce corpus fait partie d'une étude plus large réalisée dans le cadre d'une thèse de doctorat (Gehres, 2016) réalisée au laboratoire CReAAH (UMR 6566, Rennes), portant sur les problématiques liées à la production et à la diffusion de céramiques dans des contextes littoraux et insulaires sur le Massif armoricain, du Néolithique à l'Antiquité. Dans le cadre de ce travail de recherche, les méthodes employées sont similaires à celles décrites dans cet article, à savoir l'approche archéométrique des matières premières employées pour façonner les terres-cuites.

\section{ConteXte géologiQue}

La Batterie-Basse se trouve à l'extrémité nord-est du Massif armoricain, dans le Cotentin (Manche). Le site est situé dans une région où se mêlent des massifs granitiques et des roches sédimentaires (fig. 2). La côte nord du Cotentin est constituée principalement par des granites, tandis que le centre de la région est formé de roches de type schistes, grès et arkoses (Graindor, 1963; 1977). Plus au sud de la presqu'île, on notera la présence du pluton granitique de Flamanville (fig. 2), ceinturé par des roches métamorphiques (Graindor, 1963; 1977).

\section{Méthodologie}

Afin de déterminer l'origine géologique et géographique des matières premières employées pour façonner les céramiques, mais aussi d'identifier les techniques de montage, plusieurs approches multiscalaires ont été mises en œuvre à la suite des études typologiques. Les observations et analyses ont été faites, dans la mesure du possible, sur des poteries se référant à des formes connues. Nous présenterons ici les méthodes utilisées dans cette étude, empruntées pour la plupart aux Sciences de l'Univers. Pour une approche plus détaillée, on pourra se tourner vers des ouvrages de référence traitant de ce sujet (Échallier, 1984; Rice, 1987; Convertini et Querré, 1998; Quinn, 2009; 2013).

Un premier tri des poteries à l'œil nu a été réalisé selon plusieurs critères : la texture, la couleur des parois, l'abondance et la nature des éléments non plastiques, leurs tailles et leurs formes (Whitbread, 1995; Breakmans et al., 2017). La nature des cristaux est ainsi déterminée comme on procéderait pour une roche, à partir de critères pétrographiques caractéristiques à chaque espèce minérale.

Par la suite, une étude des céramiques au microscope polarisant est réalisée. Pour cela un échantillon de la poterie est préparé en lame mince. Le principe est de déterminer un maximum de critères afin d'identifier la nature des inclusions minérales présentes dans la pâte des céramiques. Ainsi, sur le Massif armoricain, il est possible d'en déduire l'ori- 
gine et la nature géologique des roches primaires, dont les potiers ont utilisé les produits d'altérations pour façonner leurs terres cuites. En effet, ce massif a la particularité d'être composé d'une grande diversité de roches (magmatiques et métamorphiques) et de peu de bassins sédimentaires. Les artisans ont donc eu accès à une multitude de terres, issues de l'altération météorique ou chimique de ces formations granitiques, gabbroïques ou encore ultrabasiques.

Ces observations fournissent également des informations sur la (ou les) technique(s) de montage, la présence ou l'absence d'une peinture, d'engobe, de graphitage des parois. L'analyse morphoscopique et granulométrique des grains permet également d'identifier des pratiques comme le tri des inclusions, le rajout d'éléments dégraissants tel que du sable, des bioclastes, des végétaux ou des os broyés.

Les terres cuites de la Batterie-Basse ont également fait l'objet d'un volet d'analyses chimiques globales par spectrométrie de fluorescence X portable (P-XRF) et ponctuelles par spectrométrie de masse à source plasma couplée à un système d'ablation laser (LA-ICP-MS).

Les analyses (P-XRF) ont été faites à l'aide d'un équipement Niton XL3t GOLDD 7, sur pastille de céramique (poudre de poterie compactée sous forme de pastille, sans adjonction de liant). La calibration de l'appareil a été réalisée à partir des étalons géologiques internationaux du Centre de Recherches Pétrographiques et Géochimiques (CRPG) : GA, GH, GS-N, MA-N, DR-N, DT-N, UB-N, MICA-Fe (Govindaraju et Roelandts, 1988 et 1989). Ces analyses nous permettent d'obtenir les concentrations des éléments chimiques majeurs $\left(\mathrm{Al}_{2} \mathrm{O}_{3}, \mathrm{SiO}_{2}, \mathrm{~K}_{2} \mathrm{O}, \mathrm{CaO}, \mathrm{TiO}_{2}\right.$, $\mathrm{MnO}_{2}, \mathrm{Fe}_{2} \mathrm{O}_{3}$ ) présents dans la terre cuite, ainsi que celles de plusieurs éléments mineurs $(\mathrm{S}, \mathrm{V}, \mathrm{Cr}, \mathrm{Zn}, \mathrm{Rb}, \mathrm{Sr}, \mathrm{Ba}, \mathrm{Pb})$. Enfin, pour les analyses LA-ICP-MS, un spectromètre de masse quadripolaire à source plasma (Agilent Technologies, 7700 Series), couplé à un système d'ablation laser Nd:YAG de 213 nm (Cetac Technologies, LSX-213, G2) a été utilisé pour réaliser des analyses chimiques ponctuelles selon la méthode développée par Gehres et Querré (Gehres et al., 2015; Gehres, 2016; Gehres et Querré, 2017; sous presse). La calibration de l'instrument a été faite à partir d'étalons géologiques internationaux : DR-N, DT-N, UB-N (Govindaraju et Roelandts, 1989) et MICA-Fe (Govindaraju et Roelandts, 1988). Au total, ont ainsi pu être dosés 46 éléments : Na, Mg, Al, Si, K, Ca, Ti, Mn, Fe, Li, Sc, V, Cr, Co, $\mathrm{Ni}, \mathrm{Cu}, \mathrm{Zn}, \mathrm{As}, \mathrm{Rb}, \mathrm{Sr}, \mathrm{Y}, \mathrm{Zr}, \mathrm{Nb}, \mathrm{Cd}, \mathrm{Sb}, \mathrm{Ba}, \mathrm{La}, \mathrm{Ce}, \mathrm{Pr}$, $\mathrm{Nd}, \mathrm{Sm}, \mathrm{Eu}, \mathrm{Gd}, \mathrm{Tb}, \mathrm{Dy}, \mathrm{Ho}, \mathrm{Er}, \mathrm{Tm}, \mathrm{Yb}, \mathrm{Lu}, \mathrm{Hf}, \mathrm{Ta}, \mathrm{Tl}$, $\mathrm{Pb}, \mathrm{Th}, \mathrm{U}$. L'obtention d'un éventail large de données nous a permis de mettre en avant la pertinence de trois éléments
Y, Dy, Eu* (* signifie que l'élément a été normalisé par rapport aux chondrites), permettant de distinguer les coquilles fossiles des coquilles contemporaines (Gehres et al., 2015; Gehres, 2016).

\section{ApProches ARCHÉOMÉTRIQUES DES MATIÈRES PREMIÈRES DES CÉRAMIQUES}

Afin de déterminer l'origine géologique et géographique des terres utilisées par les potiers pour façonner les céramiques retrouvées sur le site de la Batterie-Basse, 50 poteries ont été prélevées afin de réaliser une étude pétrographique au microscope polarisant (tabl. 1). Parmi elles, 34 ont été analysées par P-XRF pour obtenir une composition chimique globale des pâtes.

Ces résultats seront comparés à une précédente étude menée par H. Morzadec (Peuchet-Geilenbrügge et Morzadec, 2001) dont les résultats démontrent l'existence de plusieurs types de pâte (tabl. 1), classés en 8 groupes :

- Fragments de coquilles calcaires + grains de sable.

- Céramique à spicules de silicisponges et dégraissant fin homogène quartz + feldspath + micas.

- Dégraissant riche en plagioclases altérés + quartz + feldspath + micas.

- Dégraissant à chamotte + quartz + calcaire.

- Dégraissant calcaire (bryozoaires), argile limoneuse.

- Céramique micacée (biotite), quartz + feldspath + muscovite.

- Dégraissant fin à quartz dominant + petits grains de calcite.

- Dégraissant sableux essentiellement du quartz.

La réalisation de nouvelles études a été notamment motivée par la limite de ces résultats, comme le souligne A. Lefort, «si les résultats nous permettent d'esquisser quelques lignes directrices, leurs limites sont nombreuses et nous verrons que de nouvelles analyses sont fortement souhaitables " (Lefort et Marcigny, 2009, p. 67-68).

À partir des nouvelles études pétrographiques en lame mince, 7 types de pâtes ont été décrits. Ils correspondent à des assemblages minéralogiques singuliers, et sont dès lors liés à des sources de matières premières distinctes ou à des traditions techniques spécifiques, comme le rajout d'un dégraissant (le terme de dégraissant est ici employé dans le sens d'éléments rajoutés volontairement par les potiers, pour les éléments présents naturellement dans la pâte, nous préférerons le terme d'inclusions). 


\section{Les pâtes à inclusions granitiques et sédimen- taires - Groupe 1 (13 céramiques)}

Ces vases (tabl. 1) sont caractérisés par une pâte à spicules de silicisponge et fragments de roches très roulés et altérés de tailles diverses, associant des cristaux de quartz, de feldspath potassique et des tablettes de muscovite (fig. 3). Le corpus est également composé de grains de quartz, de feldspath potassique, des tablettes de muscovite et de biotite en quantité accessoire dans certaines céramiques. Des grains de feldspath plagioclase (de type albite - oligoclase) sont identifiables en plus faible quantité, tout comme des fragments de micrite et de rares cristaux d'amphibole verte et incolore. Des fragments de coquilles arrondies en faible nombre ont été observés dans tous les vases. La présence de fragments de roche de type silexoïde est à noter dans plusieurs terres cuites. Ce groupe pourrait correspondre à celui observé par H. Morzadec et caractérisé par des "Céramiques à spicules de silicisponge à dégraissant fin homogène et quartz + feldspath + micas" (Peuchet-Geilenbrügge et Morzadec, 2001). La taille maximale des inclusions atteint environ $2 \mathrm{~mm}$, et leurs tailles moyennes varient de $65 \mu \mathrm{m}$ à $100 \mu \mathrm{m}$. Les inclusions de tailles moyennes ou supérieures sont arrondies à subarrondies, et les grains les plus fins (dont la taille inférieure à $50 \mu \mathrm{m}$ est très homogène) et structurant le fond de la pâte sont anguleux à subanguleux (fig. 3).

Deux sous-groupes se distinguent (tabl. 1) par la taille plus importante des lithoclastes dans certains vases (sousgroupe $1 \mathrm{a}-7$ céramiques) et la présence plus abondante de fragments de silexoïde (sous-groupe $1 \mathrm{~b}-1$ céramique).

Ces observations indiquent que l'origine géologique des terres utilisées pour le façonnage de ces vases pourrait être un mélange de produits d'altération de roches sédimentaires et cristallines. Au niveau typologique (tabl. 1), ces pâtes ont principalement été utilisées dans le montage de jattes moyennes, et plus rarement pour celui de pots à panse globulaire, dont un enduit à l'hématite et des jattes à cordon (fig. 3). La question de l'existence d'un lien entre la forme de ces vases et l'utilisation de ce type de pâte peut être posée. Cependant, ces formes ne sont pas exclusives à ce groupe de pâte et représentent une grande partie de notre corpus, il pourrait alors s'agir d'un effet lié à l'échantillonnage. Plusieurs origines sont envisageables pour cette matière première :

- La présence en quantité plus ou moins accessoire de fragments de silexoïde nous permet de restreindre l'origine de cette matière première à la région de Flamanville, où des argiles à silex issues de la décalcification d'un étage sénonien sont connues (fig. 2). Ces gisements sont ceinturés par le granite de Flamanville, riche en amphibole (Graindor, 1977).

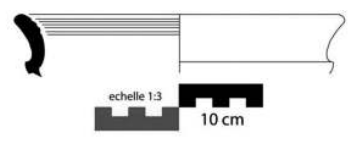

Éch. 1 - INV 012.1

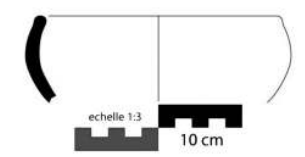

Éch. 8 - INV 011.01 / INV 2010 CER-14

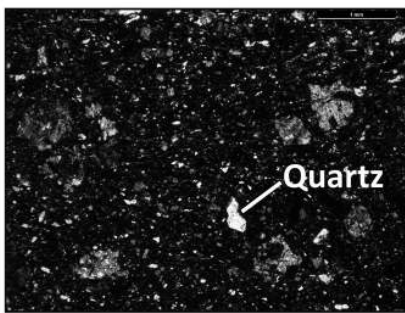

Éch. 1 - INV 012.1

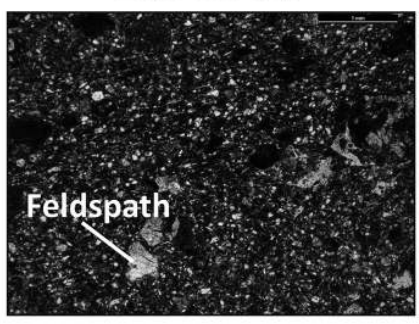

Éch. 11 - INV 021.05 / INV 2010 CER-17

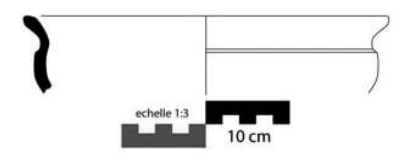

Éch. 2 - INV 014.03 / INV 2010 CER-22
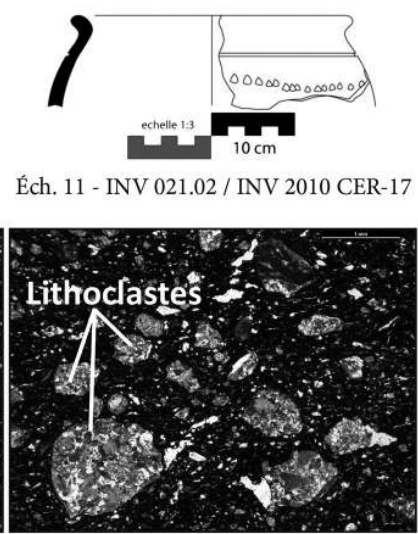

Éch. 2 - INV 014.03 / INV 2010 CER-22

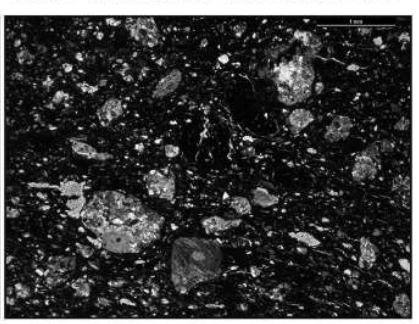

Éch. 11 - INV 021.02 / INV 2010 CER-17

Figure 3 : (Voir planche couleur XI) Typologies et micrographies des céramiques du groupe 1 . Lumière polarisée non analysée (LPNA) : éch. 1, 2 et 11; dessins : A. Lefort in Lefort, 2015.

Figure 3: (See colour plate XI) Forms and micrographs of group 1 ceramics. Natural light (LN): Smp 1, 2 and 11; drawing: A. Lefort in Lefort, 2015.

- L'utilisation d'argiles provenant d'une zone côtière, où le mélange des produits cristallins et sédimentaires se serait fait de manière naturelle. Or, les grains fins présents dans les pâtes sont pour la plupart anguleux, synonymes d'un faible transport, ce qui ne correspond pas avec un sable de plage.

\section{La pâte à inclusions gabbro-granitiques - Groupe 2 (1 céramique)}

L'abondance de cristaux anguleux d'amphibole incolore et verte très pâle caractérise la pâte de cette céramique (tabl. 1). Les autres inclusions correspondent à des grains de quartz, de feldspath potassique, de feldspath plagioclase acide et basique (de type oligoclase, andésine et labrador), ainsi que des tablettes de biotite (fig. 4). La forme très anguleuse des grains, et la taille des inclusions, qui ne dépasse pas au 


\begin{tabular}{|c|c|c|c|c|}
\hline $\begin{array}{l}\text { Groupe pétro- } \\
\text { graphique }\end{array}$ & $\mathrm{n}^{\circ}$ lame & Référence & Typologie et élément caractéristique & $\begin{array}{l}\text { Hypothèse de correspondance : Peuchet- } \\
\text { Geilenbrügge \& Morzadec, } 2001\end{array}$ \\
\hline 1 & 1 & INV 012.1 & Jatte moyenne & Groupe 2 de H. Morzadec \\
\hline 1 & 4 & INV 0.14.02 / INV 2010 CER-22 & Jatte moyenne & Groupe 2 de H. Morzadec \\
\hline 1 & 7 & INV 04.02 / INV 2010 CER-14 & Jatte moyenne & Groupe 2 de H. Morzadec \\
\hline 1 & 14 & INV 021.05 / INV 2010 CER-17 & Décor isolé & Groupe 2 de H. Morzadec \\
\hline 1 & 35 & INV 2010 CER-26 & Pot à panse globulaire & Groupe 2 de H. Morzadec \\
\hline 1a & 2 & INV 014.03 / INV 2010 CER-22 & Jatte moyenne & Groupe 2 de H. Morzadec \\
\hline 1a & 8 & INV 011.01 / INV 2010 CER-14 & Bol à panse globulaire & Groupe 2 de H. Morzadec \\
\hline 1a & 11 & INV 021.02 / INV 2010 CER-17 & Pots & Groupe 2 de H. Morzadec \\
\hline 1a & 13 & INV 021.04 / INV 2010 CER-17 & Fond & Groupe 2 de H. Morzadec \\
\hline 1a & 36 & INV 022.1 / INV 2010 CER-13 & Pot à panse globulaire & Groupe 2 de H. Morzadec \\
\hline 1a & 48 & INV 08.01 / INV 2010 CER-4 & Jatte à cordons & Groupe 2 de H. Morzadec \\
\hline 1a & 49 & INV 08 / INV 2010 CER-4 & Décor isolé & Groupe 2 de H. Morzadec \\
\hline $1 \mathrm{~b}$ & 25 & INV 19.03 / INV 2012 CER-20 & Bord à décor isolé & Groupe 2 de H. Morzadec \\
\hline 2 & 3 & INV 014-05 / INV 2010 CER-22 & Fond & 1 \\
\hline 3 & 5 & INV 014.01 / INV 2012 CER-22 & Jatte moyenne & Groupe 1 de H. Morzadec \\
\hline 3 & 6 & INV 014-04 / INV 2010 CER-22 & Jatte moyenne & Groupe 1 de H. Morzadec \\
\hline 3 & 9 & INV 011.03 / INV 2010 CER-14 & Bord & Groupe 1 de H. Morzadec \\
\hline 3 & 12 & INV 021.03 / INV 2010 CER-17 & Bord & Groupe 1 de H. Morzadec \\
\hline 3 & 16 & INV 021.07 / INV 2010 CER-17 & Bord & Groupe 1 de H. Morzadec \\
\hline 3 & 18 & INV 020.01 / INV 2010 CER-19 & Bol / Jatte à collerette & Groupe 1 de H. Morzadec \\
\hline 3 & 37 & INV 022.3 & Jatte à cordons & Groupe 1 de H. Morzadec \\
\hline 3 & 45 & INV 2010 CER-6 & Bord & Groupe 1 de H. Morzadec \\
\hline 3 & 46 & INV 2010-CER6 & Jatte à collerette & Groupe 1 de H. Morzadec \\
\hline $3 \mathrm{a}$ & 19 & INV 0.20.02 / INV 2010 CER-19 & Pieds de vase balustre & Groupe 1 de H. Morzadec \\
\hline $3 \mathrm{a}$ & 41 & INV 05 / INV 2010 CER-9 & Bol / Jatte à collerette & Groupe 1 de H. Morzadec \\
\hline $3 \mathrm{~b}$ & 15 & INV 021.06 / INV 2012 CER-17 & Panse à décor isolé & Groupe 1 de H. Morzadec \\
\hline $3 b$ & 23 & INV 019.015 / INV 2010 CER-20 & Bord & Groupe 1 de H. Morzadec \\
\hline $3 \mathrm{~b}$ & 27 & INV 019.05 / INV 2010 CER-20 & Pot à panse globulaire & Groupe 1 de H. Morzadec \\
\hline $3 \mathrm{~b}$ & 28 & INV 19.09 / INV 2010 CER-20 & Panse à décor isolé & Groupe 1 de H. Morzadec \\
\hline $3 \mathrm{~b}$ & 38 & INV 022.4 & Jatte moyenne & Groupe 1 de H. Morzadec \\
\hline $3 \mathrm{~b}$ & 39 & INV 022.02 / INV 2010 CER-13 & Jatte moyenne & Groupe $1 \mathrm{de} \mathrm{H}$. Morzadec \\
\hline $3 \mathrm{~b}$ & 40 & INV 022.05 / INV 2010 CER-13 & Vase balustre & Groupe 1 de H. Morzadec \\
\hline $3 \mathrm{~b}$ & 43 & INV 07 / INV 2010 CER-2 & Jatte moyenne & Groupe 1 de H. Morzadec \\
\hline $3 \mathrm{~b}$ & 50 & Sd 23 & Décor isolé & Groupe 1 de H. Morzadec \\
\hline $3 c$ & 26 & INV 19.10 / INV 2010 CER-20 & Jatte à cordon & Groupe 1 de H. Morzadec \\
\hline $3 c$ & 30 & INV 19.08 / INV 2012 CER-20 & Pied de vase balustre & Groupe 1 de H. Morzadec \\
\hline $3 c$ & 31 & INV 19.02 / INV 2010 CER-20 & Jatte moyenne & Groupe 1 de H. Morzadec \\
\hline $3 c$ & 32 & INV 19.12 / INV 2010 CER-20 & Pot & Groupe 1 de H. Morzadec \\
\hline $3 c$ & 33 & INV 19.07 / INV 2010 CER-20 & Gobelet & Groupe 1 de H. Morzadec \\
\hline $3 c$ & 34 & INV 017.01 / INV 2010 CER-15 & Jatte à collerette & Groupe 1 de H. Morzadec \\
\hline 4 & 47 & INV 02 / INV 2010 CER-8 & Pot & Groupe 5 de H. Morzadec \\
\hline 5 & 10 & INV 021.01 / INV 2010 CER-17 & Jatte moyenne & 1 \\
\hline 6 & 17 & INV 21.08 / INV 2010 CER-17 & Pied de vase balustre & Groupe 3 de H. Morzadec \\
\hline 6 & 21 & INV 013.01 / INV 2010 CER-21 & Pieds de jatte moyenne & Groupe 3 de H. Morzadec \\
\hline 6 & 22 & INV 019.01 / INV 2012 CER-20 & Jatte moyenne & Groupe 3 de H. Morzadec \\
\hline 6 & 24 & INV 19.17 / INV 2010 CER-20 & Pied de vase balustre & Groupe 3 de H. Morzadec \\
\hline 6 & 44 & INV 2010 CER-6 & Bord & Groupe 3 de H. Morzadec \\
\hline 7 & 20 & INV 015.01 / INV 2010 CER-23 & Pot & Groupe 8 de H. Morzadec \\
\hline 7 & 29 & INV 19.06 / INV 2010 CER-20 & Bord à décor isolé & Groupe 8 de H. Morzadec \\
\hline $7 \mathrm{a}$ & 42 & INV 01 / INV 2012 CER-10 & Gobelet & Groupe 8 de H. Morzadec \\
\hline
\end{tabular}


maximum $900 \mu \mathrm{m}$, pour une taille moyenne de $150 \mu \mathrm{m}$ (fig. 4), sont selon nous des arguments allant dans le sens d'un broyage de la matière première par les potiers avant son utilisation. Cette terre correspond à l'altération d'une roche basique à cristaux d'amphibole de type gabbro et d'un granite. Son origine se trouve dans les zones d'ateliers de potier de Saint-Jean-du-Doigt (Finistère) et de Trégomar (Ille-et-Vilaine), connues pour produire des céramiques à pâte gabbro granitique (Giot et Querré, 1987; Morzadec, 1995). Des analyses chimiques ponctuelles par LA-ICP-MS des grains d'amphibole ont permis de confirmer que cette terre cuite provient de la zone de production de SaintJean-du-Doigt. En effet, les deux ensembles d'amphibole se différencient au travers de plusieurs éléments majeurs : $\mathrm{MgO}, \mathrm{Al}_{2} \mathrm{O}_{3}, \mathrm{Fe}_{2} \mathrm{O}_{3}$ (Finistère; Gehres, 2016; Gehres et Querré, 2017; sous presse). Cette approche s'appuie sur une méthode mise en place dans nos précédentes recherches, basée sur la comparaison des signatures chimiques des minéraux (ici les amphiboles) présents dans la pâte de la poterie et des cristaux de la même nature inclus dans les roches mères supposées être à l'origine des matières premières utilisées (Gehres, 2016; Gehres et Querré, 2017; sous presse).

\section{Les pâtes à inclusions coquillères rajoutées - Groupe 3 (26 céramiques)}

Ce type de pâte est le plus représenté dans le corpus étudié sur le site de la Batterie-Basse (tabl. 1). Il se singularise par la présence en grande quantité de fragments anguleux de coquillages peu altérés (fig. 5). Les autres inclusions identifiées correspondent à des grains principalement anguleux, mais également subarrondis de quartz et de feldspath potassique. Des bioclastes arrondis sont plus rarement perceptibles tout comme des fragments roulés de micrite (fig. 5). La catégorisation dans cet article entre coquilles fossiles et contemporaines a été réalisée dans un premier temps à partir des observations pétrographiques en lame mince. En effet, l'absence de fragments de calcaire libres dans la pâte ou cimentés aux coquilles est le critère principal permettant de distinguer la nature des bioclastes. La morphologie des grains est principalement anguleuse et quelques arrondis sont décelables. La taille moyenne des inclusions minérales varie de $100 \mu \mathrm{m}$ à $310 \mu \mathrm{m}$ et leur taille maximale est de

Tableau 1 : (page précédente) Les céramiques du second âge du Fer étudiées, du site de la Batterie-Basse (Urville-Nacqueville, Manche) - Typologie et groupes pétroarchéologiques.

Table 1: (prévious page) Late Iron Age ceramics studied from the site of La Batterie-Basse (Urville-Nacqueville, Manche) - Forms and petroarchaeological groups.

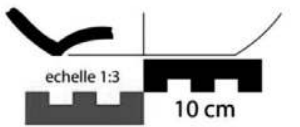

Éch. 3 - INV 014-05 / INV 2010 CER-22

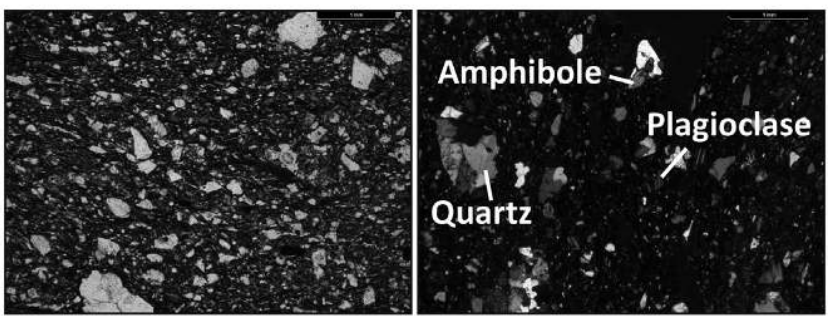

Éch. 3 - INV 014-05 / INV 2010 CER-22

Figure 4 : (Voir planche couleur XII) Typologie et micrographies de la céramique du groupe 2. LPNA : éch. 3, micrographie de gauche; Lumière polarisée (LP) : éch. 3, micrographie de droite; dessins : A. Lefort in Lefort, 2015.

Figure 4: (See colour plate XII) Forms and micrographs of group 2 ceramic. LN: Smp 3, micrograph on the left; Polarised light (LP): Smp 3, micrograph on the right; drawing: A. Lefort in Lefort, 2015.

$1 \mathrm{~mm}$ (fig. 5). On remarquera que les grains de quartz et de feldspath potassique possèdent des tailles situées dans une fourchette restreinte, pouvant être un indice allant dans le sens d'un tri ou d'un broyage de ces inclusions avant incorporation dans la matière première. Il s'agit probablement d'une terre issue de l'altération d'une roche granitique, en partie épurée puis dégraissée à partir de fragments de coquilles broyées. Une telle matière première est accessible aux alentours du site, où affleurent des granites (Graindor, 1977). Ce type de terre semble correspondre à celui décrit par $\mathrm{H}$. Morzadec dans son étude des vases à inclusions de "fragments de coquilles calcaires + grains de sable" (PeuchetGeilenbrügge et Morzadec, 2001).

Dans cet ensemble, il a été possible de discerner trois sousgroupes, qui sont basés sur la variation d'espèces minérales. La présence de coquilles anguleuses y est toujours attestée en grand nombre. Le sous-groupe 3 a (2 céramiques) est composé de céramiques dont la pâte a été épurée et présente des inclusions de quartz et de feldspath potassique le plus souvent bien triées, accompagnées par des tablettes de muscovite et de biotite. La sous-catégorie $3 \mathrm{~b}$ ( 9 céramiques) est minéralogiquement proche du sous-groupe précédent, bien qu'ils s'y ajoutent de très rares fragments de calcaire, mais aussi des grains d'amphibole verte de manière accessoire. Le sous-ensemble 3c (6 céramiques) se singularise par la présence en grande proportion de fragments anguleux de silexoïdes. Les autres inclusions minérales sont les mêmes que celles observées dans le premier sous-groupe 1a. 


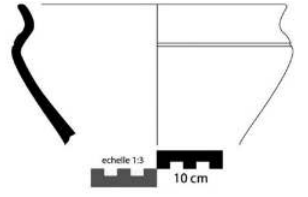

Éch. 5 -INV 014.01 / INV 2012 CER-22

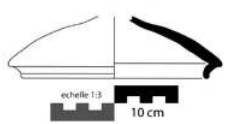

Éch. 18 - INV 020.01 / INV 2010 CER-19

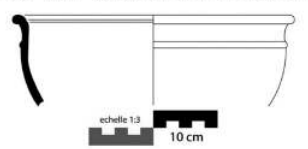

Éch. 37 - INV 022.3

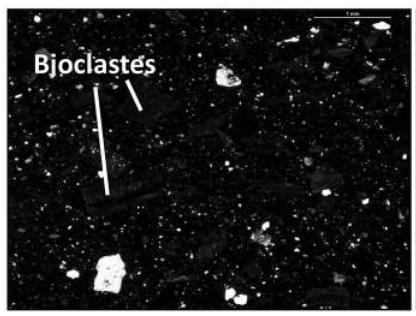

Éch. 37 - INV 022.3

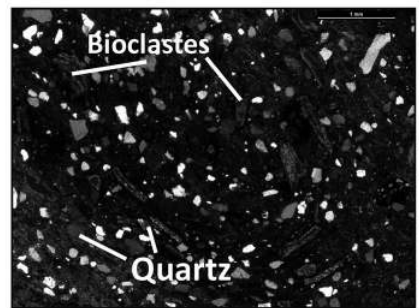

Éch. 23 - INV 019.015 / INV 2010 CER-20 Éch. 30 - INV 19.08 / INV 2012 CER-20

Figure 5 : (Voir planche couleur XII) Typologies et micrographies des céramiques du groupe 3. LP : éch. 37, 41 et 23; LPNA : éch. 30; dessins : A. Lefort in Lefort, 2015.

Figure 5: (See colour plate XII) Forms and micrographs of group 3 ceramics. LP: Smp 37, 41 and 23; LN: Smp 30; drawing: A. Lefort in Lefort, 2015.

Létude de ces différentes sous-catégories de pâte démontre la diversité de la nature des inclusions présentes dans les terres de ces poteries, laissant supposer des origines diversifiées pour ces matières premières. En effet, les deux premiers sous-groupes correspondent à une terre d'origine granitique, qui aurait pu provenir des altérations des granites proches du site (Graindor, 1977). Les pâtes de la dernière sous-catégorie correspondent à une argile à silex. Plusieurs zones peuvent avoir fourni une telle matière première : les argiles à silex présentes sur le granite de Flamanville (Graindor, 1977) à environ $20 \mathrm{~km}$ au sud du site (fig. 2), ou encore les terrasses marines du Haut Normannien contenant des galets de silex roulés dans la région de Vrasville, Gouberville et Montfarville (fig. 2) à une vingtaine de kilomètres au sud-est de la Batterie-Basse (Graindor et Pareyn, 1969).

Les tailles moyennes et maximales des fragments de coquillages frais, et des inclusions minérales ont été mesurées. On observe une calibration de la taille des bioclastes. En effet, la taille moyenne des coquilles est comprise entre 0,4 et $0,5 \mathrm{~mm}$, et les tailles maximales dépassent rarement 1,5 mm. La standardisation des tailles moyennes et maximales et l'angularité des fragments de coquilles tendent à prouver que les potiers du site de la Batterie-Basse cherchaient à obtenir une taille spécifique de coquille broyée avant de les incorporer dans l'argile.

Au niveau typologique, on remarque l'utilisation de ce type de dégraissant et de pâte pour façonner plusieurs types de vases (fig. 5). En effet, des poteries ayant pu servir à la consommation ou la présentation de boisson comme des gobelets, ou des vases balustres ont été façonnées à partir d'argiles dégraissées à la coquille, tout comme des poteries de présentation ou de conservation de nourriture, comme des jattes ou pots. Il ne paraît donc pas exister de spécifications dans l'utilisation de ces argiles, on remarque toutefois qu'aucun de ces vases ne semble avoir été graphité ou enduit d'hématite. Il pourrait donc s'agir ici de la volonté des potiers de laisser nues ces céramiques afin que l'on puisse voir leur dégraissant.

\section{La céramique de la plaine de Caen - Groupe 4 (1 céramique)}

Ce vase a été monté à partir d'une terre où sont présentes des coquilles et des fragments de calcaire micritique encore cimentés à des tests (tabl. 1). Contrairement au groupe précédent, les coquilles ne sont pas broyées et certaines sont encore visibles quasi entières sur les cassures du tesson (fig. 6).

Ces coquilles sont associées à de rares grains de feldspath potassique et de quartz. Il semble donc s'agir d'inclusions fossilifères, comme le montre la cimentation des coquilles à des fragments de calcaire. Ce type de pâte semble avoir été décrit par H. Morzadec dans une précédente étude et pourrait correspondre au type à « dégraissant calcaire (bryozoaires) et argile limoneuse " (Peuchet-Geilenbrügge et Morzadec, 2001).

Cette céramique a été façonnée à partir d'une marne ou d'une argile de décalcification d'un calcaire coquillier. Cette roche est connue dans la plaine de Caen (fig. 2), tout comme des céramiques à pâte fossilifère dites "productions de la plaine de Caen" (San Juan et al., 1999a, 1999b; Carpentier et al., 2002; Besnard-Vauterin et al., 2009; Jahier et al., 


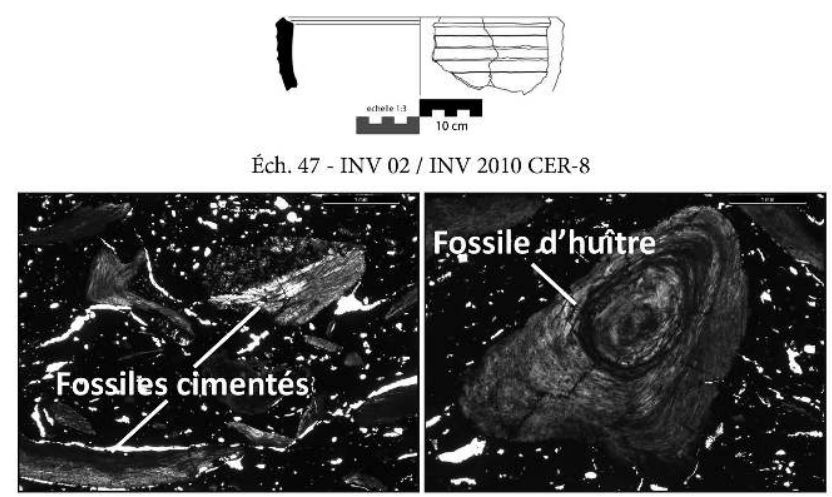

Éch. 47 - INV 02 / INV 2010 CER-8

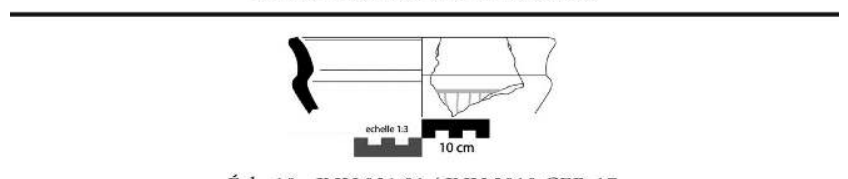

Éch. 10 - INV 021.01 / INV 2010 CER-17

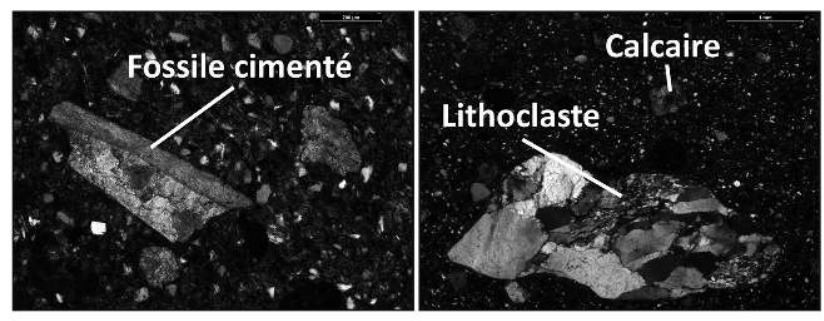

Éch. 10 - INV 021.01 / INV 2010 CER-17

Figure 6 : (Voir planche couleur XIII) Typologies et micrographies des céramiques des groupes 4 et 5. LP : éch. 47 et 10 ; dessins : A. Lefort in Lefort, 2015.

Figure 6: (See colour plate XIII) Forms and micrographs of groups 4 and 5 ceramics. LP: Smp 47 and 10; drawing: A. Lefort in Lefort, 2015.

2011; Manson et al., 2011). Il s'agit donc d'une importation de cette région. Cette hypothèse est cohérente avec la forme de cette terre cuite. En effet, il s'agit d'un pot tronconique typique des "productions de la plaine de Caen" dont une comparaison typologique est réalisable sur le site de Mondeville, dans la région de Caen, vase qui avait été analysé pétrographiquement par $\mathrm{H}$. Morzadec qui a pu mettre en évidence les mêmes types d'inclusions que dans la poterie découverte à la Batterie-Basse (Peuchet-Geilenbrügge et Morzadec, 2001).

\section{La céramique à pâte fossilifère - Groupe 5 (1 céramique)}

Une jatte moyenne a été montée à partir d'une terre provenant manifestement de la décalcification d'un calcaire coquillier (tabl. 1), comme le démontre la présence de nombreux fragments de calcaire associés à des bioclastes fossiles (fig. 6). Des grains de micrite sont dis- séminés dans la pâte ainsi que des grains de tourmaline bleue cimentés à des fragments de calcaire. Cet assemblage minéralogique n'a pas été décrit par $\mathrm{H}$. Morzadec. Contrairement à la céramique du groupe 4 , dont les caractéristiques des productions de la plaine de Caen sont clairement visibles (fossiles quasi entiers, aucun tri, gros fragments de calcaire coquillier), cette jatte présente des inclusions fines et un fond fin de grains triés, ainsi que des morceaux de calcaire coquillier plus petit et plus fragmenté. Ces inclusions sont majoritairement anguleuses, mais présentent également des côtés émoussés, leurs tailles varient en moyenne de $100 \mu \mathrm{m}$ à $220 \mu \mathrm{m}$ tandis que la taille maximale atteint $4 \mathrm{~mm}$ (fig. 6). L'assemblage minéralogique des inclusions présentes dans cette céramique permet d'avancer plusieurs hypothèses quant à son origine :

- Il pourrait s'agir d'un vase issu des " productions de la plaine de Caen ". L'emploi d'une argile de décalcification plutôt que d'une marne à calcaire fossile (ou inversement) pourrait expliquer les nuances observées entre cette terre cuite et celle du groupe précédent.

- Il serait possible qu'il s'agisse d'une production importée des îles britanniques. Ce type de pâte a effectivement pu être observé dans plusieurs céramiques du site d'Hengistbury Head, sans, toutefois, correspondre exactement à notre description (Cunliffe et al., 1987).

\section{Les pâtes à inclusions granitiques - Groupe 6 (5 céramiques)}

Les pâtes de cet ensemble possèdent des inclusions correspondant à des produits d'altération d'une roche granito gneissique (tabl. 1). Il s'agit de grains de quartz, de feldspath potassique, de tablettes de micas (principalement de la biotite puis de la muscovite), ainsi que des cristaux de plagioclase (de type oligoclase). La particularité de ce groupe réside dans la présence de grains de microcline en grande quantité dans la majorité des vases (fig. 7). Les inclusions sont majoritairement anguleuses à subarrondies. Leurs tailles varient en moyenne de $100 \mu \mathrm{m}$ à $220 \mu \mathrm{m}$ tandis que la taille maximale atteint $4 \mathrm{~mm}$ (fig. 7). Aucun tri ou broyage des grains ne semble avoir eu lieu. Ce groupe pourrait correspondre aux vases à "dégraissant riche en plagioclases altérés + quartz + feldspath + micas» identifiés par H. Morzadec (Peuchet-Geilenbrügge et Morzadec, 2001).

L'hypothèse d'une production d'origine locale semble la plus probable. De nombreux granites formant une partie du socle du Cotentin sont constitués partiellement de cristaux de microcline (fig. 2), notamment les granites de Gréville ou d'Auderville (Graindor 1977). Typologiquement, ces pâtes 


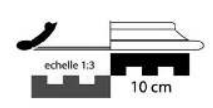

Éch. 24 - INV 19.17 / INV 2010 CER-20

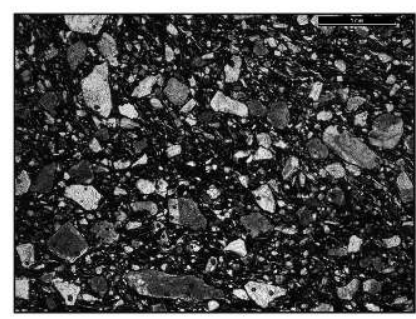

Éch. 17 - INV 21.08 / INV 2010 CER-17

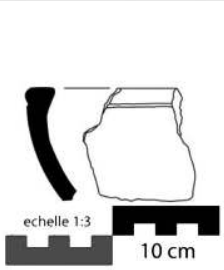

Éch. 20 - INV 015.01 / INV 2010 CER-23

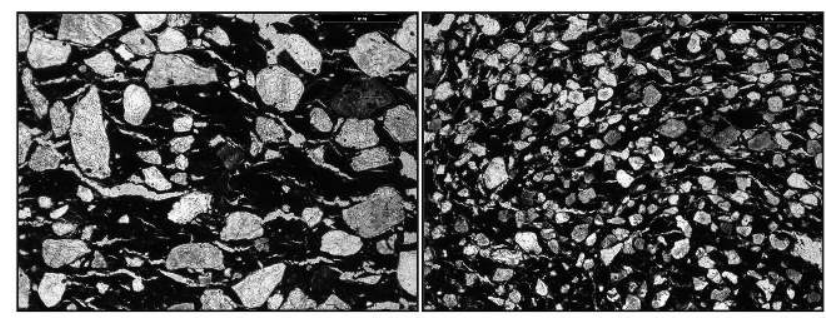

Éch. 20 - INV 015.01 / INV 2010 CER-23 Éch. 42 - INV 01 / INV 2012 CER-10

Figure 7 : (Voir planche couleur XIV) Typologies et micrographies des céramiques des groupes 6 et 7. LPNA : éch. 17, 20 et 42; LP : éch. 44; dessins : A. Lefort in Lefort, 2015.

Figure 7: (See colour plate XIV) Forms and micrographs of groups 6 and 7 ceramics. LN: Smp 17, 20 and 42; LP: Smp 44 drawing: A. Lefort in Lefort, 2015.

ont principalement servi à façonner des vases balustres ainsi que des jattes moyennes (fig. 7).

\section{Les pâtes à dégraissant sableux - Groupe 7 (3 céramiques)}

Ces terres ont été dégraissées à partir de sable bien trié (tabl. 1). Ce groupe pourrait correspondre à celui identifié par H. Morzadec comme étant l'ensemble à "dégraissant sableux, essentiellement du quartz " (Peuchet-Geilenbrügge et Morzadec, 2001). L'étude des inclusions de ces vases indique qu'il s'agit de grains de quartz majoritairement et de feldspath potassique. En quantité accessoire, sont identifiables de très petits fragments de silexö̈de (fig. 7). Les inclusions sont arrondies à subarrondies. La taille moyenne des grains est d'environ $360 \mu \mathrm{m}$ et la taille maximale observée de $1 \mathrm{~mm}$ (fig. 6). Ces observations ont montré que ces grains sont très bien triés. Il apparaît donc que les artisans ont épuré leurs terres afin de les dégraisser à l'aide de sable bien calibré. La présence de petits fragments de coquillages roulés plaide plutôt en faveur d'un sable de plage (fig. 7).

Une céramique (sous-groupe 7a) se distingue par l'utilisation d'un sable fin et subarrondi. On notera notamment l'absence de fragments de calcaire, de micrite, de coquillage et l'existence d'éléments arrondis ferrugineux. La taille moyenne des inclusions est comprise en 170 et $400 \mu \mathrm{m}$, tandis que la taille maximale est de $1 \mathrm{~mm}$. L'origine des inclusions de la pâte de la céramique du sous-groupe $7 \mathrm{a}$ semble être la même que celle des grains de la poterie du groupe 7 :

- L'emploi de produits d'altération d'un grès ferrugineux, dont des affleurements sont connus dans la région d'Urville-Nacqueville (Graindor, 1977).

- Le rajout d'un sable dunaire dans une pâte épurée.

Les grains présents dans cette céramique sont le plus souvent subarrondis, forme qui rappelle celles des sables de type RM (rond mat) issus de l'érosion éolienne. La seconde hypothèse serait donc la plus probable.

Ce type de pâte est associé à un pot ou bol et un bord droit évoquant la forme d'une jatte, et à un gobelet de bonne taille (fig. 7).

L'emploi de sable comme dégraissant peut être considéré comme risqué du fait d'un coefficient de dilation du quartz supérieur à celui des argiles (Gibson et Woods, 1990; Rye, 1976; Woods, 1986), la poterie perdant en résistance et en solidité à chaque chauffe. Cependant, comme l'ont montré les récents travaux de N. Muller et al. "Quartz, which at $573{ }^{\circ} \mathrm{C}$ undergoes a reversible phase transition accompanied by a 7 vol \% change, is frequently cited in discussions of strength reduction. However, the volume fractions of the quartz component in both temper types, as determined by XRD methods, are quite similar, reasonably low (0.26 for granite and 0.19 for phyllite), and cannot account on their own for the observed differences" (Muller et al., 2010, p. 2460). La question de l'épuration de ces pâtes et du rajout d'un dégraissant sableux pour les céramiques de la Batterie-Basse est dès lors difficile à expliquer. Malgré cela, on peut remarquer que ces trois céramiques n'ont probablement pas été destinées à être chauffées, puisqu'il s'agit de pot, de jatte et de gobelet. Les potiers ont pu alors délibérément choisir de façonner des céramiques dont l'utilisation ne relèvera pas, par la suite, de la cuisson, mais plutôt du service ou du stockage, sans toutefois que ce geste ne soit lié à la présentation au feu des vases. Leurs origines restent toutefois très compliquées 
à déterminer puisque peu d'éléments minéralogiques caractéristiques sont observables.

\section{LES ANALYSES CHIMIQUES DES CÉRAMIQUES DU SITE DE LA BATTERIE-BASSE}

Des analyses chimiques globales par P-XRF ont été réalisées sur 34 vases (tabl. 2). Les céramiques à pâtes granitiques (groupe 6) se distinguent par leurs concentrations en $\mathrm{K}_{2} \mathrm{O}$ et $\mathrm{Al}_{2} \mathrm{O}_{3}$ qui sont nettement plus élevées (tabl. 2), confirmant la distinction entre les poteries à inclusions granitiques et sédimentaires (groupe 1). La céramique à pâte à inclusions gabbroïques se distingue des autres vases par de fortes teneurs en $\mathrm{Fe}_{2} \mathrm{O}_{3}, \mathrm{Al}_{2} \mathrm{O}_{3}, \mathrm{Cr}, \mathrm{V}$, Cu et un déficit de la concentration en $\mathrm{K}_{2} \mathrm{O}$, plus présent pour les poteries dont les terres proviennent de l'altération de roches granitiques. Le vase importé de la Plaine de Caen (groupe 4) possède une teneur plus élevée en $\mathrm{CaO}$ (tabl. 2). On peut expliquer ce phénomène par la plus faible dilution de cet élément dans l'analyse globale, du fait de l'absence d'éléments granitiques. En comparant ces résultats à ceux de la céramique à pâte fossilifère (groupe 5), on remarque que la composition globale de cette dernière dénote fortement de celle de la plaine de Caen (groupe 4). En effet, les différentes concentrations nous indiquent bien qu'il s'agit d'une poterie dont l'origine géologique est différente de la terre cuite typique de la plaine de Caen. Les teneurs des éléments comme le $\mathrm{SiO}_{2}, \mathrm{Al}_{2} \mathrm{O}_{3}$ et $\mathrm{K}_{2} \mathrm{O}$, précédemment faibles, sont ici moyennes et comprises entre celles de pâtes fossilifères et celles de pâtes granitiques (tabl. 2). Il s'agit donc bien d'un mélange entre ces deux types de roches. Il en va de même pour les céramiques à pâte bioclastique (groupe 3) et à pâte à inclusions granitiques et sédimentaires (groupe 1). En effet, ces pâtes semblent posséder des caractéristiques chimiques proches, comprises entre celles de la pâte fossilifère (groupe 5) et celles des vases gra- nitiques (groupe 6). Ces observations nous indiquent qu'il s'agît bien de céramiques dont les inclusions correspondent à des assemblages minéralogiques granitiques et des éléments calciques. On remarque cependant des différences entre les poteries du groupe 3 et du groupe 1 . En effet, les céramiques à bioclastes sont légèrement plus concentrées en $\mathrm{CaO}$, tandis que celles à inclusions granitiques et sédimentaires ont des teneurs plus élevées en $\mathrm{SiO}_{2}$ (tabl. 2). Les terres cuites à coquilles rajoutées pourraient avoir été montées à partir des argiles à inclusions granitiques et sédimentaires préalablement épurées. Enfin, les céramiques à pâte sableuses possèdent des concentrations élevées en $\mathrm{SiO}_{2}$, du fait de l'importante présence du quartz dans la pâte.

\section{L'ANALYSE CHIMIQUE PONCTUELLE PAR LA-ICP-MS DES INCLUSIONS DE COQUILLAGES FOSSILES ET CONTEMPORAINS}

L'utilisation d'argile de décalcification de calcaire coquillier ou de marnes à fossiles est attestée sur de nombreux sites, comme celui de Chalain (Martineau et al., 2007), ou encore en plaine de Caen (San Juan et al., 1999a et 1999b; Carpentier et al., 2002; Besnard-Vauterin et al., 2009; Manson et al., 2011). Il s'agit dans ce cas d'une argile où sont naturellement présentes des inclusions de coquillages fossiles. D'autres sites ont quant à eux permis d'observer des vases dégraissés à partir de coquilles qui n'étaient pas présentes naturellement dans les terres. Il peut s'agir alors de fossiles comme sur le site de La Perroche (Guiavarc'h et Querré, 2009), ou d'éléments contemporains des artisans, ramassés sur l'estran ou dans les rivières pour la consommation et/ou leur adjonction à la pâte comme sur le site des Gouillauds (Rousseau et al., 2001). Ils sont alors préalablement passés au feu, afin de les rendre plus faciles à broyer (Bronitsky et Hammer, 1986; Rice, 1987). Cependant, il n'est pas toujours

\begin{tabular}{|c|c|c|c|c|c|c|c|c|c|c|c|c|c|c|c|c|c|}
\hline & $\begin{array}{l}\text { Nbre de } \\
\text { céramique } \\
\text { analysée }\end{array}$ & $\begin{array}{c}\mathrm{Al}_{2} \mathrm{O}_{3} \\
\%\end{array}$ & $\sigma$ & $\begin{array}{c}\mathrm{SiO}_{2} \\
\%\end{array}$ & $\sigma$ & $\begin{array}{c}\mathrm{K}_{2} \mathrm{O} \\
\%\end{array}$ & $\sigma$ & $\begin{array}{c}\mathrm{CaO} \\
\%\end{array}$ & $\sigma$ & $\begin{array}{c}\mathrm{TiO}_{2} \\
\%\end{array}$ & $\sigma$ & $\begin{array}{c}\mathrm{MnO}_{2} \\
\%\end{array}$ & $\sigma$ & $\mid \begin{array}{c}\mathrm{Fe}_{2} \mathrm{O}_{3} \\
\%\end{array}$ & $\sigma$ & $\begin{array}{c}\mathrm{Cr} \\
\mathrm{ppm}\end{array}$ & $\sigma$ \\
\hline Groupe 1 & 8 & 20,6 & 0,88 & 62,6 & 3,44 & 2,6 & 0,64 & 5,3 & 2,08 & 0,8 & 0,33 & 0,1 & 0,03 & 6,2 & 1,44 & 173 & 75,99 \\
\hline Groupe 2 & 1 & 28,1 & 1 & 50,2 & 1 & 1,2 & 1 & 4,8 & 1 & 0,8 & 1 & 0,1 & 1 & 10,7 & 1 & 526 & 1 \\
\hline Groupe 3 & 15 & 21,4 & 2,98 & 56,6 & 3,39 & 2,6 & 0,29 & 10,3 & 5,35 & 0,8 & 0,14 & 0,1 & 0,02 & 5,8 & 1,42 & 135 & 21,19 \\
\hline Groupe 4 & 1 & 17,2 & 1 & 43,5 & 1 & 3,2 & 1 & 27,7 & 1 & 0,7 & I & 0,1 & 1 & 6,4 & I & 95 & 1 \\
\hline Groupe 5 & 1 & 20,1 & 1 & 56,7 & 1 & 2,5 & 1 & 7,6 & 1 & 0,5 & 1 & 0,3 & 1 & 9,0 & 1 & 149 & I \\
\hline Groupe 6 & 5 & 25,9 & 2,59 & 57,6 & 2,1 & 3,9 & 1,08 & 3,2 & 1,85 & 0,8 & 0,12 & 0,1 & 0,03 & 4,7 & 0,78 & 109 & 15,53 \\
\hline Groupe 7 & 3 & 19,2 & 1,75 & 66,6 & 3,06 & 2,2 & 0,27 & 3,7 & 2,68 & 0,5 & 0,08 & 0,1 & 0,01 & 6,1 & 2,58 & 96 & 57,93 \\
\hline
\end{tabular}

Tableau 2 : Moyennes et écarts-types des éléments majeurs des différents groupes de pâtes de céramiques obtenus par P-XRF. Table 2: Average concentrations and standard deviations of the major elements from the different groups of ceramic pastes obtained by P-XRF. 
aisé de distinguer la nature fossilifere ou contemporaine des bioclastes inclus dans les terres, notamment lorsque les deux types de pâtes sont présents sur le site. Afin d'identifier la nature des coquilles, une approche par analyses chimiques ponctuelles par LA-ICP-MS a été développée (Gehres et al., 2015; Gehres, 2016; Gehres et Querré, 2017). Au niveau des éléments majeurs, on remarque une relative homogénéité entre les coquilles fossiles et les contemporaines, tandis que les teneurs en $\mathrm{Li}, \mathrm{Cr}, \mathrm{Sr}, \mathrm{Y}$ et $\mathrm{Ba}$ ainsi que celles des terres rares légères ( $\mathrm{La}, \mathrm{Ce}, \mathrm{Pr}, \mathrm{Nd}, \mathrm{Sm}, \mathrm{Eu}, \mathrm{Gd}$ ) sont plus hétérogènes et permettent de distinguer les fossiles des coquilles fraîches. On remarque ainsi que sur le diagramme binaire $\mathrm{Eu}^{*} / \mathrm{Dy}^{*}$ les analyses correspondant aux coquilles fossiles et aux coquilles contemporaines sont localisées autour de deux droites ayant des coefficients directeurs différents (fig. 8). Il existe donc une relation de proportionnalité entre le $\mathrm{Dy}$ et l'Eu* au sein des coquilles. Sur les diagrammes ternaires $\left(\mathrm{Dy}^{*}, \mathrm{Eu}^{*}, \mathrm{Y}\right)$ et $\left(\mathrm{Dy}^{*}\right.$, $\mathrm{Eu}^{*}, \mathrm{Th}$ ) il est possible de distinguer clairement deux groupes (fig. 9). L'un représentant les inclusions fossiles et l'autre les coquilles contemporaines. On remarque enfin que les coquillages frais possèdent des teneurs plus élevées en terres rares légères que les fossiles analysés.

\section{DisCUSSIONS DES RÉSULTATS}

\section{Incorporation de coquilles broyées et chaîne opératoire}

Au vu du nombre de céramiques dégraissées à partir de coquilles broyées, il est possible de supposer que les potiers devaient avoir une quantité importante de broyat afin de préparer un volume suffisant d'argile pour monter plusieurs séries de vase. Ceci démontrerait qu'il ne s'agit pas d'actes opportunistes, mais plutôt de préparations réfléchies, faisant partie intégrante de la chaîne opératoire. De fait, celle-ci s'en trouve rallongée par les différentes étapes nécessaires à la préparation des coquilles avant de pouvoir être rajoutées. Ainsi, après avoir été collectées, pour la consommation puis l'utilisation dans les céramiques (ou vides pour être directement préparées), il est probable que les coquilles aient été passées au feu, afin de les déshydrater et d'enlever les fractions organiques pouvant encore y être accrochées (Carter, 2002), mais également pour les rendre plus faciles à broyer (Bronitsky et Hammer, 1986; Rice, 1987). Le rajout de coquille comme dégraissant est une pratique sujette à discussion et de nombreuses explications ont été avancées. Elles peuvent être mécaniques, comme le montrent les travaux de V. Steponaitis sur la résistance des poteries à bioclastes aux chocs thermiques et aux stress mécaniques.
Ce dernier démontre l'utilisation de gros fragments de coquilles pour dégraisser des terres serviront à monter des poteries allant au feu, et l'emploi de petits fragments pour les vaisselles de présentation de la nourriture (Steponaitis, 1983, 1984). Cependant, ce rajout peut aussi répondre à des problématiques plus abstraites, liées à une tradition culturelle et cultuelle. On citera les travaux de J. Feathers sur les céramiques à dégraissant coquillier dans le golfe du Mexique, qui voit aussi dans ces pratiques la volonté d'améliorer la résistance des vases, mais aussi du fait de sa perduration sur au moins un millénaire, une raison plus traditionnelle (Dunnels et Feathers, 1991; Feathers, 1989, 2006, 2009; Feathers et Peacock, 2008; Feathers et Scott, 1989). D'autres chercheurs avancent le fait qu'il pourrait s'agir plutôt d'une méthode permettant de diminuer la plasticité de certaines argiles comme les montmorillonites. Le $\mathrm{CaO}$ (chaux vive) qui apparaît alors pendant la cuisson des coquilles (selon l'équation $\mathrm{CaCO}_{3} \rightarrow \mathrm{CaO}+\mathrm{CO}_{2}$ ) permettrait de réduire la quantité d'eau présente dans l'argile, la rendant ainsi plus malléable, la formation de $\mathrm{Ca}(\mathrm{OH})_{2}$ produirait également des ions de calcium qui entrainerait la floculation de la pâte et améliorerait sa plasticité (Stimmel et al., 1982). Cependant, la transformation des coquilles en chaux, qui se déroule à partir de $700-800{ }^{\circ} \mathrm{C}$, peut fragiliser par la suite des récipients, par la réhydratation de la chaux qui va augmenter son volume (Rye, 1976, 1981).

Néanmoins, il est possible de pallier ce problème de plusieurs manières :

- Maintenir la température de cuisson en dessous du seuil de transformation (Hammond, 1971; Rye, 1976; Steponaitis, 1983; Tankersley et Meinhart, 1982; Vitelli, 1984), ce qui signifierait la pratique d'une cuisson en tas.

- Atteindre une température supérieure à $1000^{\circ} \mathrm{C}$, afin de faire fusionner l'argile et le calcium (Maniatis et Tite, 1981 ; Rice, 1987). Cela nécessiterait dès lors de pratiquer une cuisson en four.

- Mélanger à la pâte une faible quantité de sel (Klemptner et Johnson, 1986; Rye 1976; Stimmel et al., 1982).

On peut supposer que les potiers du site de la BatterieBasse ont employé une de ces solutions, selon les différents degrés de technologie à leur disposition. Ces observations nous permettent d'extrapoler plusieurs hypothèses quant aux raisons du rajout de coquilles dans les argiles des céramiques du site de la Batterie-Basse. En effet, on peut remarquer qu'il n'existe pas de lien entre la taille des coquilles pillées (groupe 3), qui est en moyenne plutôt constante et la forme ainsi que l'utilisation de ces poteries : consommation ou contenant de boisson (gobelets, vases balustres), présentation ou conservation de nourriture (jattes, pots). 
Figure 8 : Diagramme binaire représentant les concentrations en $\mathrm{Eu}^{*}$ et Dy des coquilles inclues dans les céramiques. Chaque point correspond à l'analyse par LA-ICP-MS d'un fragment de coquille (d'après Gehres et al., 2015; Gehres, 2016 et Gehres et Querré, 2017).

Figure 8: Binary diagram of Eu* and Dy concentrations from tempered crushed shells. Each point corresponds to an analysis by LA-ICP-MS of one crushed shell (after Gehres et al., 2015; Gehres, 2016 and Gehres and Querré, 2017).

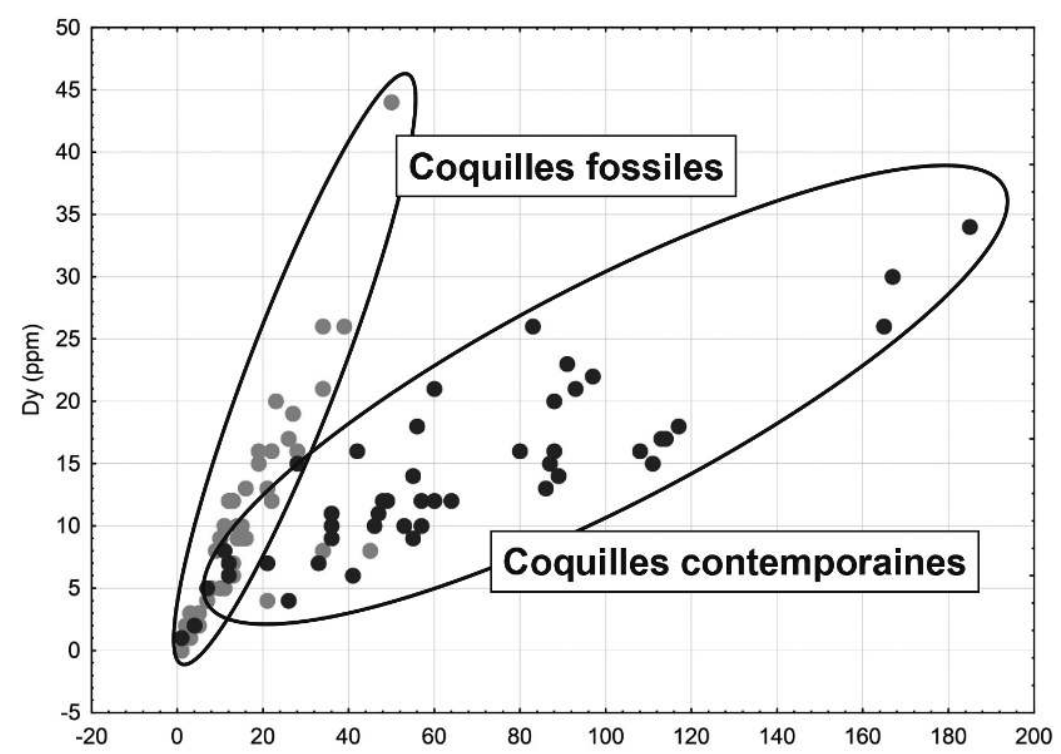

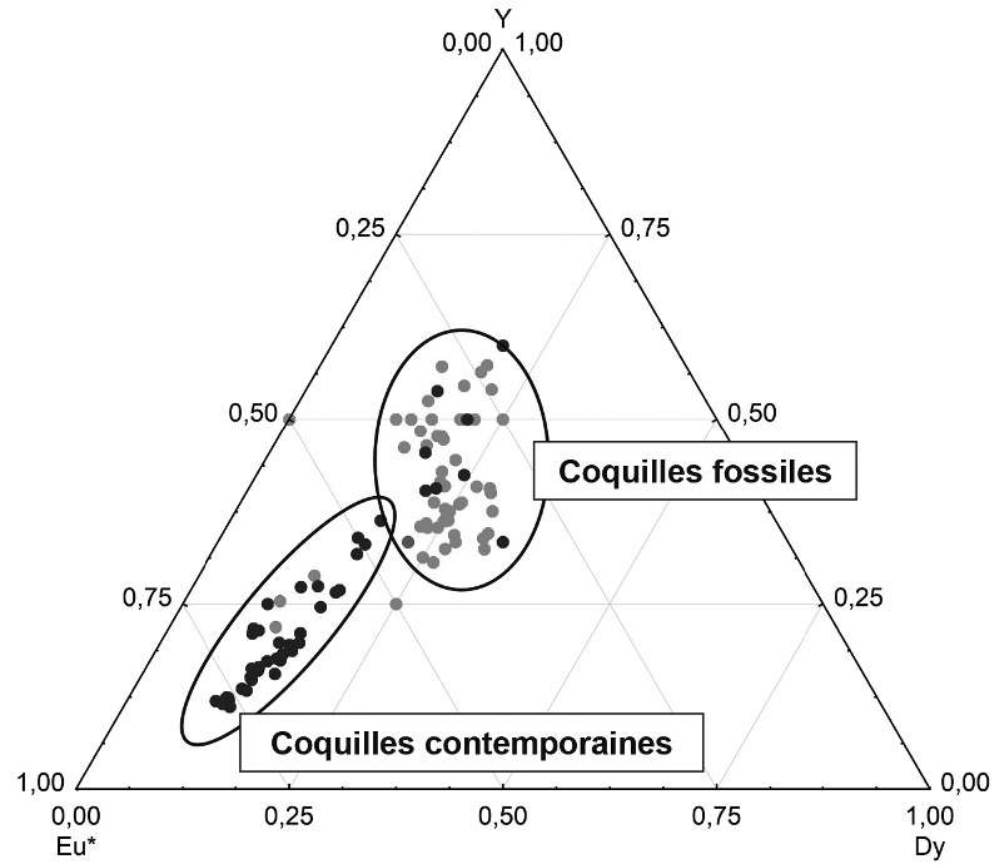

Figure 9: Diagramme ternaire représentant les concentrations en Eu*, Dy et $\mathrm{Y}$ des coquilles inclues dans les céramiques. Chaque point correspond à l'analyse par LA-ICP-MS d'un fragment de coquille (d'après Gehres et al., 2015; Gehres, 2016 et Gehres et Querré, 2017).

Figure 9: Ternary diagram of Eu*, Dy and $Y$ concentrations from tempered crushed shells. Each point corresponds to an analysis by LA-ICP-MS of one crushed shell (after Gehres et al., 2015; Gehres, 2016 and Gehres and Querré, 2017).
Ainsi, l'hypothèse d'un rajout de coquilles en vue d'améliorer les résistances des poteries ne semble pas correspondre aux volontés des divers potiers du site de la Batterie-Basse. Cependant, nous ne pouvons pas éliminer entièrement cette hypothèse. Toutefois, la question du remplacement de ce type de dégraissant par l'utilisation de chamotte à la place peut se poser, notamment du fait d'un raccourcissement de la chaîne opératoire. La réponse se trouverait alors dans les conclusions de J. Feathers, qui voit dans cette adjonction une pratique culturelle et cultuelle.
Enfin, on peut citer le rajout de coquilles fossiles (lamellibranche, cardium, huître, scrobiculaire) issus des niveaux crétacés et du cénomanien inférieur, et broyées dans la pâte des céramiques du Néolithique final du site de la Perroche sur l'île d'Oléron (Guiavarc'h et Querré, 2009).

\section{Des échanges entre le site de la Batterie-Basse et le sud de l'Angleterre}

De nombreux indices découverts sur ce site permettent de mieux appréhender les échanges ayant pu avoir lieu entre le 
nord Cotentin et le sud de l'Angleterre. Ainsi, des études sur les bracelets et ébauches en lignites retrouvés dans ce gisement permettent d'envisager une origine anglaise de la matière première, la reliant aux affleurements de lignite de Kimmeridge, confirmant ainsi les hypothèses de P.-R. Giot (Giot et al., 1986) : notamment l'analyse palynologique de J.B. Riding, non publiée, mais souvent citée (Paris et al., 2000; Le Nagard, 2005; Marcigny et Ghesquière, 2005; Marcigny et al., 2007), ou encore les travaux de A. Baron (Baron, 2012). Les fouilles de Nacqueville ont également permis de mettre au jour des particularités architecturales au travers de bâtiments à plan circulaire qui renvoient aux traditions britanniques de construction et plutôt rares sur le continent (Lefort, 2015; Lefort et Marcigny, 2009; Lefort et al., 2015). Enfin, la fouille de la nécropole a mise au jour des corps inhumés en position recroquevillée (Lefort et al., 2015), rappelant les « crouched burials» durotriges connus à la même époque en Angleterre (Cunliffe, 2005; Papworth, 2008; Fitzpatrick, 2010).

Du point de vue des céramiques, en plus d'une anse à œillet rattachée aux productions durotriges par L. Brown (Lefort et Marcigny, 2009), des céramiques "fines à cordons " appelées également "black cordoned wares" ont été découvertes sur le site. Elles correspondent notamment à des marqueurs d'échanges entre l'Armorique et l'Angleterre (Daire, 1992) et sont très présentes sur le site d'Hengistbury Head (Wheeler et Richardson, 1957, Cunliffe et al., 1987). Deux poteries ont été identifiées comme des «saucepan pots ", forme absente du continent et typique du Wessex. La première poterie a été montée à partir d'une pâte sableuse (sous-groupe 7a). L'origine de ces grains nous semblait être dunaire, sans pouvoir préciser l'origine géographique de ce vase. Au vu de sa forme, cette origine pourrait être britannique. La seconde céramique correspond à une pâte à inclusions coquillères (sous-groupe 3c), dont l'origine semble locale au vu du nombre de vases ayant une signature pétrographique et chimique proche. Ainsi, ces deux terres cuites présentent une forme inconnue sur le continent, mais bien connue dans le Wessex. Leurs pâtes diffèrent fortement et ne sont pas comparables. L'une pourrait avoir été produite en Angleterre tandis que l'autre parait être le produit d'une assimilation de la forme des "saucepan pots" sur le site d'Urville-Nacqueville. Un pot (fig. 3; éch. 8) ne trouve également pas de comparaison dans l'ouest de la Gaule, mais uniquement en Angleterre, tout comme le pot dont le décor se retrouve sur un vase d'Hengistbury Head (comm. personnelle A. Lefort). Ces deux céramiques font partie du groupe 1a, caractérisé par des lithoclastes sédimentaires et magmatiques. Dans ce cas, une origine locale de la matière première et des céramiques semble la plus probable. Enfin, comme nous avons pu le voir précédemment, le rajout de coquilles pilées dans la pâte des céramiques ne paraît pas être un processus lié à un renforcement des poteries. Une des possibilités avancées serait qu'il puisse s'agir d'un phénomène empreint d'une tradition culturelle ou d'actes liés à des pratiques cultuelles. Aucun pendant de ce côté de la Manche n'est pour l'instant connu et nous devrons nous tourner vers le sud de l'Angleterre, où sur le site d'Hengistbury Head ou du Camp Cambourne de tels rajouts paraissent avoir été observés (Cunliffe et al., 1987; Wright et al., 2009). Cependant, les auteurs de ces études pétrographiques ne précisent pas s'il s'agit de rajout de coquilles contemporaines ou de coquilles fossiles déjà présentes ou adjointes à la pâte comme c'est le cas sur des sites datés du Néolithique et de l'âge du Bronze ancien du Wessex (Cleal, 1995).

\section{Conclusions}

L'étude de la matière première des terres cuites de la zone artisanale de la Batterie-Basse à Urville-Nacqueville a permis de mettre en avant l'existence de plusieurs types de pâte sur ce site. La plupart de ces vases semblent provenir de la région, ce qui démontre une bonne implantation de l'occupation et une bonne connaissance des gisements de terre. On remarque ainsi l'utilisation de ressources comme des terres granitiques (groupe 6) issues de l'altération du socle régional, mais aussi de matière première provenant de zones plus éloignées comme les terres à inclusions granitiques et sédimentaires (groupe 1), dont l'origine reste à préciser, oscillant entre les argiles à silex du massif granitique de Flamanville ou une argile d'estran ayant accumulé les divers cristaux issus de l'altération du contexte géologique local. Des pâtes plus atypiques ont également été observées, il s'agit notamment des céramiques à dégraissant sableux (groupe 7). L'origine de ces vases reste pour l'instant difficilement localisable. Il pourrait s'agir d'une production locale, faite sur l'instant, pour pallier le manque de dégraissant coquillier, comme d'une importation d'un site plus spécialisé dans ce type de vase.

Des importations sont présentes sur le site comme un vase typique des "productions de la plaine de Caen" (groupe 4), dont une poterie, dont la forme et la pâte sont similaires à celle de la Batterie-Basse, a été découverte sur le site de Mondeville dans la région de Caen (Peuchet-Geilenbrügge et Morzadec, 2001). L'origine non locale d'une jatte façonnée à partir d'altérations de calcaire coquillier (groupe 5) différentes de celles des "productions de la plaine de Caen " est confirmée sans toutefois pouvoir être précisée. Il pourrait s'agir d'une importation anglaise, ou d'une poterie montée à partir d'une matière première localisée dans la région de 
Caen, mais dont les caractéristiques minéralogiques sont différentes des "productions de la plaine de Caen». Un vase (groupe 2), a été identifié comme issu de la zone de production de céramique à pâte gabbro granitique de SaintJean-du-Doigt (Gehres et Querré, 2017; sous presse), soit à plus de $180 \mathrm{~km}$ d'Urville-Nacqueville par voie maritime. La présence à Nacqueville de ce vase démontre le rayonnement qu'a pu avoir cet atelier durant le second âge du Fer.

On remarque donc un faible taux de céramiques importées sur le site de la Batterie-Basse, contrairement aux attentes que l'on aurait pu avoir lorsque l'on fait la liste des produits importés découverts sur le site. Ce phénomène pourrait s'expliquer par le fait que nos analyses ne portent que sur les poteries de la zone artisanale du site, il serait donc nécessaire de continuer à étudier les céramiques qui seront découvertes dans la suite des fouilles de la nécropole, située à proximité de la zone artisanale.

L'analyse des céramiques du site de la Batterie-Basse à Urville-Nacqueville apporte de nouveaux éléments à la problématique des échanges transmanche au second âge du Fer. Il apparaît au vu des nombreuses découvertes faites sur ce site que ces échanges ne se limitaient pas à des pratiques commerciales, mais bien à des transferts technologiques et culturels (Giot et al., 1986; Baron, 2012; Lefort, 2015; Lefort et Marcigny, 2009; Lefort et al., 2015), comme la pratique issue d'outre-Manche de l'ajout de bioclastes dans les pâtes, dont il n'existe pas, en l'état actuel de la recherche, de comparaison dans l'ouest de la Gaule pour cette époque. Ce faisceau d'indices de plus en plus dense au fur et à mesure des fouilles et de l'analyse des artefacts du site de la BatterieBasse nous démontre l'existence de contacts et d'échanges entre le Cotentin et le sud de l'Angleterre, clairement établis par ailleurs (Cunliffe et De Jersey, 1997; Cunliffe, 2001; Lefort, 2015), suffisamment nombreux et fréquents pour permettre aux occupants de ce site l'assimilation de pratiques architecturales, artisanales et funéraires.

\section{Bibliographie}

Baron A., 2012. Provenance et circulation des objets en roches noires "lignite "à l'âge du Fer en Europe celtique (VIII-I - siècle av. J.C.). British Archaeological Report, International series 2453, Oxford.

Besnard-Vauterin C.-C., Savary X, Sehier E. et Zaour N., 2009. La culture matérielle, un reflet de la vie domestique et agropastorale. In C.-C. Besnard-Vauterin (dir.). En plaine de Caen, une campagne gauloise et antique. L'occupation du site de l'Étoile à Mondeville. Rennes, Presses Universitaires de Rennes, p. $87-133$.
Braekmans D., Degryse P., Neyt B., Waelkens M., Poblome J., 2017. Reconstructing Regional Trajectories: the Provenance and Distribution of Archaic to Hellenistic Ceramics in Central Pisidia (South-west Turkey), Archaeometry, 59, p. 472-492.

Bronitsky G., Hammer R., 1986. Experiments in Ceramic Technology: The Effects of Various Tempering Materials on Impact and Thermal-Shock Resistance. American antiquity, 51, (1), p. 89-101.

Carpentier V., Marcigny C., Savary X., avec la collaboration de GHesquière E., 2002. Enclos et souterrain du second Âge du Fer dans la Plaine de Caen, l'exemple de Cormelles-le-Royal (Calvados). Revue Archéologique de l'Ouest, 19, p. 37-60.

Carter M., 2002. Prehistoric Ceramic Production: Raw Materials and Firing Methods of the La Crosse Locality Oneota. Thèse de doctorat, Université du Wisconsin, États-Unis.

Cleal R., 1995. Pottery Fabric in Wessex in the Fourth to the Second Millenia B.C. In I. Kinnes, G. Varndell (eds.). Unbaked Urns of Rudely Shape: Essayes on British and Irish Pottery from Ian Longworth. Oxbow, Oxford, p. 185-194.

Convertini F., Querré G., 1998. Apports des études céramologiques en laboratoire à la connaissance du Campaniforme : résultats, bilan et perspectives. Bulletin de la Société prébistorique française, 95, (3), p. 333-342.

Cunliffe B., 2001. Facing the Ocean. The Atlantic and its Peoples 8000 B.C.-AD 1500. Oxford University Press, Oxford.

Cunliffe B., 2005. Iron Age Communities in Britain. An Account of England, Scotland and Wales from the Seventh Century B.C. until the Roman Conquest. Routledge, Londres.

Cunliffe B., Brown L., Barton R.N.E., 1987. Hengistbury Head, Dorset. The Prehistoric and Roman Settlement, 3500BCAD500. Oxford, Oxford University School of Archaeology, Oxford University Committee for Archaeology Monograph.

Cunliffe B., De Jersey P., 1997. Armorica and Britain: CrossChannel Relationships in the Late First Millennium B.C. Oxford, Studies in Celtic Coinage, Oxford University Committee for Archaeology Monograph.

DAIRE M.-Y., 1992. Les céramiques armoricaines à la fin de l'âge du Fer, Travaux du Laboratoire d'Anthropologie. Travaux du Laboratoire d'Anthropologie, Université Rennes 1.

Dunnel R., Feathers J., 1991. Late Woodland Manifestations of the Malden Plain, Southeast Missouri. In M. Nassaney, C. Cobb (eds.). Stability, Transformation, and Variation: the Late Woodland Southeast. New York, p. 21-45.

ÉCHAllier J.-C., 1984. Éléments de technologie céramique et d'analyse des terres cuites archéologiques. Documents d'archéologie méridionale, numéro spécial, Série méthodes et techniques, 3. Association pour la diffusion de l'archéologie méridionale, Lambesc. 
Feathers J., 1989. Effects of Temper on Strength of Ceramics: Response to Bronitsky and Hamer. American Antiquity, 54, (3), p. 579-588.

Feathers J., 2006. Explaining Shelltempered Pottery in Prehistoric Eastern North America. Journal of Archaeological Method and Theory, 13, (2), p. 89-133.

Feathers J., 2009. Problems of Ceramic Chronology in the Southeast: Does Shelltempered Pottery Appear Earlier Than we Think? American Antiquity, 74, (1), p. 113-142.

Feathers J., Peacock E., 2008. Origins and Spread of ShellTempered Ceramics in the Eastern Woodlands: Conceptual and Methodological Frameworks for Analysis. Southeastern Archaeology, 27, (2): 286-293.

Feathers J., Scott W. D., 1989. Prehistoric Ceramic Composite from Mississippi Valley. American Ceramic Society Bulletin, 68, (3), p. 554-557.

Fitzpatrick A., 2010. Les pratiques funéraires de l'Âge du Fer tardif dans le sud de l'Angleterre. In P. Barral, B. Dedet, F. Delrieu, P. Giraud, I. Le Goff, S. Marion, A. Villars-Le Tiec (dir.). Gestes funéraires en Gaule au Second Âge du Fer. Actes du XXXIII ${ }^{\mathrm{e}}$ colloque de l'Association Française pour l'Étude de l'âge du Fer, Caen, 2009, Presses Universitaires de FrancheComté, Besançon, p. 15-30.

Gehres B., 2016. Connaissances des sociétés insulaires armoricaines par l'étude archéométrique du mobilier céramique. Les réseaux d'échanges îles-continent : évolution du Néolithique à la période gallo-romaine. Thèse de doctorat, Université de Rennes 2.

Gehres B., Querré G., 2017. La signature chimique des inclusions minérales comme traceur de l'origine des céramiques : l'apport des analyses par LA-ICP-MS. In L. Burnez-Lanotte (dir.). Matières à Penser : Raw Materials Acquisition and Processing in Early Neolithic Pottery Productions, Actes du workshop de Namur (Belgique), 29 et 30 mai 2015, Séances de la Société préhistorique française, 11, p. 177-197.

Gehres B., Querré G., sous presse. New Applications of LA-ICP-MS for Sourcing Archaeological Ceramics: MicroAnalysis of Inclusions as Finger-Prints of Their Origin, Archaeometry.

Gehres B., Querré G., SAvary X. avec la collaboration de Lefort A., 2015. Caractérisation des céramiques à bioclastes de la Protohistoire dans l'Ouest de la France. In C. Mougne, M.-Y. Daire (eds.). L'Homme ses ressources et son environnement à l'âge du Fer dans le Nord-Ouest de la France, Actes du Séminaire Archéologique de l'Ouest. Nantes, France. Rennes : Mémoire de Géoscience Rennes, 9, p. 105-119.

Gibson A., Woods A.J., 1990. Prehistoric Pottery for the Archaeologist. Leicester University Press, Leicester.

Giot P.-R., Daire M.-Y., Querré G., 1986. Un habitat protohistorique : le Moulin de la Rive en Locquirec (29). Travaux du Laboratoire d'Anthropologie, Rennes.
Giot P.-R., Querré G., 1987. Premiers apports de la pétroarchéologie à l'étude des poteries préhistoriques et protohistoriques armoricaines. Bulletin de la Société Préhistorique Française, 84, (6), p. 177-182.

Govindaraju K., Roelandts I., 1988. Compilation Report (1966-1987) on Trace Elements in five CRPG Geochemical Reference Samples: Basalt BR; Granites, GA and GH; Micas, Biotite Mica-Fe and Phlogopite Mica-Mg. Geostandards Newsletter, 12, p. 119-201.

Govindaraju K., Roelandts I., 1989. 1988 compilation report on trace elements in six ANRT rock reference samples: diorite DR-N, serpentine UB-N, bauxite BX-N, disthene DT-N, granite GS-N and potash feldspar FK-N. Geostandards Newsletter, 13, p. 5-67.

Graindor M.-J., 1963. Carte géologique de France (1/50000), feuille Cherbourg, 72, BRGM, Orléans.

Graindor M.-J., 1977. Notice explicative, carte géologique de France (1/50000), feuille Cherbourg, 72, BRGM, Orléans.

Graindor M.-J., Pareyn C., 1969. Notice explicative, carte géologique de France (1/50000), feuille Saint Vaast-La Hougue, 73, BRGM, Orléans, 13 p.

Guiavarc'H M., Querré G., 2009. Les céramiques du Néolithique final de l'île d'Oléron - Études en laboratoire. In L. Laporte (dir.). Des premiers paysans aux premiers métallurgistes sur la façade atlantique (3500-2000 av. J.-C.). Association des Presses Chauvinoises, Mémoire 33, Chauvigny, p. 329-340.

Hammond P.C., 1971. Ceramic technology of South-West Asia, Syro-Paslestine: Iron IIB, Hébron. Science and Archaeology, 5, p. 11-21.

Jahier I., Savary X, Yvinec J.-H., Ghesquière E., 2011. Le mobilier. In I. Jahier (dir.). Entre résidence aristocratique et place de collecte monumentale. L'enceinte des premiers et seconds âges du Fer de La Fosse Touzé (Courseulles-sur-Mer, Calvados). Document d'archéologie française, 104. Maison des sciences de l'Homme (MSH), Paris, p. 111-146.

Klemptner L.J., Johnson P.E., 1986. Technology and the Primitive Potter: Mississippian Pottery Development Seen Throught the Eyes of a Ceramic Engineer. In W. D. Kingery (dir.)., Ceramic and Civilization, Technology and Style, 2. The American Ceramic Society, Colombus, p. 251-271.

Le Nagard H., 2005. Le mobilier en lignite de l'Âge du Fer dans le massif armoricain et ses marges. Les Dossiers $d u$ Centre Régional d'Archéologie d'Alet, 33, p. 55-78.

Lefort A. avec la collaboration de Morin O., 2012. Notes sur les monnaies gauloises découvertes sur le site d'Urville-Nacqueville (Manche). In C. Marcigny (dir.), Archéologie, histoire et anthropologie de la presqu'île de La Hague (Manche). Études et travaux, 6, p. 23-37.

Lefort A., 2015. Contribution bas-normande à une archéologie des relations transmanche à la fin de l'âge du Fer. Échanges maté- 
riels et culturels, Thèse de doctorat de l'université de Bourgognel Franche-Comté, p. 631.

Lefort A., Marcigny C., 2009. La probable agglomération portuaire du second âge du Fer d'Urville-Nacqueville, un état de la documentation. Bulletin de l'Association Manche Atlantique pour la Recherche Archéologique dans les Îles, 22, p. 39-81.

Lefort A., Rottier S., Baron A., Blondel F., Meniel P., avec la collaboration de Gratuze B., Nieto S., 2015. Artisanat, échanges et nécropole : un port de commerce de La Tène D1 à Urville-Nacqueville (Nord-Cotentin)? In F. Olmer, R. Roure, P. Barral, P. Gruat, M.-P. Roulière-Lambert, L. Pernet, Girard B. et C. Jorda (dir.). Les Gaulois au fil de l'eau, Actes du XXXVII colloque international de l'Association française pour l'étude de l'âge du Fer, Montpellier, 20-24 mai 2009, Presses Universitaires de Franche-Comté, p. 481-514.

Manson A.-L., Savary X., Cherel A.-F., 2011. Typologie et pétrographie des productions céramiques de la Plaine de Caen au premier et second âge du Fer. In P. Barral, B. Dedet, F. Delrieu, P. Giraud, I. Le Goff, S. Marion, A. Villard-Le Tiec (dir.). L'âge du Fer en Basse-Normandie, Actes du XXXIII colloque international de l'Association Française pour l'Étude de l'âge $d u$ Fer, Caen, 20-24 mai 2009. Besançon, Presses universitaires de Franche-Comté, p. 231-246.

Marcigny C., Ghesquière E., 2005. L'âge du Fer dans la Hague : le site d'Urville-Nacqueville. In C. Marcigny (dir.) et E. Ghesquière (coll.). Archéologie, histoire et anthropologie de la presqu'île de la Hague (Manche). Analyse sur la longue durée d'un espace naturel et social cohérent, première année de recherche. Beaumont-la-Hague, p. 109-114.

Marcigny C., Ghesquière E., Kinnes I., 2007. Bronze Age Cross-Channel Relations. The Lower-Normandy (France). Example: Ceramic Chronology and First Reflections. In C. Burgess, P. Topping, F. LYNCH (dir.). Beyond Stonehenge, essays on the Bronze Age in honnour of Colin Burgess. Oxbow Books, p. 255-267.

Martineau R., Walter-Simonnet A.-V., Grobéty B., Buatier M., 2007. Clay Resources and Technical Choices for Neolithic Pottery (Chalain, Jura, France): Chemical, Mineralogical and Grain-Size Analyses. Archaeometry, 49, (1), p. 23-52.

Maniatis Y., Tite M. S., 1981. Technological Examination of Neolithic Bronze Age Pottery From Central and Southeast Europe and From the Near East. Journal of Archaeological Science, 81, (1), p. 59-76.

Morzadec H., 1995. Pétro-archéologie des céramiques armoricaines du Néolithique à l'Âge du Fer. Travaux du Laboratoire d'Anthropologie de Rennes, p. 41.

Papworth M., 2008. Deconstructing the Durotriges. A definition of Iron Age communities within the Dorset environs. Oxford, British Archaeological Reports, British Series 462.
Paris P., Petit C., Huault V., Pradier B., Faggionato J.-L., 2000. Le vase en sapropélite de Saint-Gatien-des-Bois (La Tène D1, Calvados, Basse-Normandie) témoin d'échange entre la Bretagne insulaire et la Gaule. In M. Tuffreau-Libre, A. Jacques (dir.). La céramique en Gaule et Bretagne romaines: commerce, contacts et romanisation, Actes de la Table Ronde d'Arras, 23-25 octobre 1998. Centre de Recherche Archéologiques et de Diffusion Culturelle, Berck-sur-Mer, Nord-Ouest Archéologie, 12, p. 107-116.

Peuchet-Geilenbrügge C., Morzadec H., 2001. The Iron Age in Lower Normandy: a Definition Through Ceramics. In J. Collis (dir.). Society and Settlement in Iron Âge Europe, Actes du XVIII colloque de l'Association Française pour l'Étude de l'âge du Fer. Winchester 1994, p. 190-229.

Quinn P. S., 2009. Interpreting Silent Artefacts, Petrographic Approaches to Archaeological Ceramics. Archaeopress, Oxford.

QuinN P. S., 2013. Ceramic Petrography: the Interpretation of Archaeological Pottery and Related Artefacts in Thin Section. Archaeopress, Oxford.

Rice P., 1987. Pottery Analysis, a Sourcebook. Chicago, The University of Chicago Press.

Rousseau J., Robert P., Cantin N., Dupont C. avec la collaboration de Gruet Y., Huet N., Perrichot V., 2001. Exploitation d'un territoire insulaire au Néolithique moyen d'après la fouille du dépôt coquillier les Gouillauds à BoisPlage-en-Ré, (Charente-Maritime, France). Revue d'archéométrie, 25, p. 225-242.

Rye O.S., 1976. Keeping Your Temper Under Control: Materials and the Manufacture of Papuan Pottery. Archaeology and Physical Anthropology in Oceania, 11, (2), p. 106-137.

Rye O.S., 1981. Pottery Technology: Principles and Reconstruction. Washington D.C., Taraxacum.

San Juan G., Savary X., Gasnier M., 1999a. La poterie au temps des Gaulois l'exemple du site de Thaon. In G. San Juan, J. Maneuvrier (dir.). L'exploitation ancienne des roches dans le Calvados: Histoire et Archéologie, p. 361-367.

San Juan G., Meniel P., Matterne-Zeck V., Savary X., Jardel K., avec la contribution de Forfait, N. et Jahier, I., 1999 b. L'occupation gauloise au nord-ouest de Caen. L'évaluation en sondage du plateau de Thaon (Calvados). Revue Archéologique de l'Ouest, 16, p. 131-194.

Steponaitis V., 1983. Ceramic, Chronology and Community Patterns-An Archaeological Study at Moundville. New York, Academic Press.

Steponaitis V., 1984. Technological Studies of Prehistoric Pottery From Alabama: Physical Properties and Vessel Function. In S.E Van Der Leeuw, A. Pritchard (dir.). The Many Dimensions of Pottery: Ceramics in Archaeology and Anthropology. Amsterdam, Université d'Amsterdam, p. 79-122. 
Stimmell C., Heimann R.B., Hancock R.G.V., 1982. Indian Pottery From the Mississipi Valley: Coping With Bad Raw Material. In J.S. Olin, A. D. Franklin (dir.). Archaeological Ceramics. Washington D. C., Smithsonian Institution Press, p. 219-228.

Tankersley K., Meinhart J., 1982. Physicial and Structural Properties of Ceramic Materials Utilized by a Fort Ancient Group. Midcontinental Journal of Archaeology, 7, (2), p. 225-243.

Vitelli K.D., 1984. Greek Neolithic Pottery by Experiment. In P.M. Rice (dir.). Pots And Potters, Institute of Archaeology. Los Angeles, Monograph 24, p. 113-211.

WheEler R.E.M, Richardson K., 1957. Hillforts of Northern France, Report of the Research. Committee of the Society of Antiquaries of London, 19, Londres.
Whitbread I. K., 1995. Greek Transport Amphorae: A Petrological and Archaeological Study. Fitch Laboratory Occasional Paper, 4, Athens, British School at Athens.

Woods A.J., 1986. Form, Fabric, and Function: Some Observations on the Cooking Pot in Antiquity. In W.D. Kingery (dir.). Ceramic and Civilization, Technology and Style, 2, Columbus, The American Ceramic Society, p. 157-172.

Wright J., Leivers M., Seager-Smith R., Stevens C., avec la contribution de Allen M., Andrews P., Barnett C., Brown K., Gale R., Hamilton-Dyer S., Hayward K., Jones G. P., Mckinley J., Scaife R., Wells N., Wyles F., 2009. Cambourne New Settlement Iron Age and Romano-British Settlement on the Clay Uplands of West Cambridgeshire. Wessex archaeology report, p. 23. 